\title{
A rigorous derivation of mean-field models describing 2D micro phase separation
}

\author{
Barbara Niethammer ${ }^{1} \cdot$ Yoshihito Oshita $^{2}$
}

Received: 9 July 2019 / Accepted: 31 December 2019 / Published online: 15 February 2020

(c) The Author(s) 2020

\begin{abstract}
We study the free boundary problem describing the micro phase separation of diblock copolymer melts in the regime that one component has small volume fraction $\rho$ such that the micro phase separation results in an ensemble of small disks of one component. We consider the two dimensional case in this paper, whereas the three dimensional case was already considered in Niethammer and Oshita (Calc Var PDE 39:273-305, 2010). Starting from the free boundary problem restricted to disks we rigorously derive the heterogeneous mean-field equations on a time scale of the order of $\mathcal{R}^{3} \ln (1 / \rho)$, where $\mathcal{R}$ is the mean radius of disks. On this time scale, the evolution is dominated by coarsening and stabilization of the radii of the disks, whereas migration of disks becomes only relevant on a larger time scale.
\end{abstract}

\section{Mathematics Subject Classification 35B27 $\cdot 35 \mathrm{R} 35 \cdot 82 \mathrm{D} 60$}

\section{Introduction}

Diblock copolymer molecules consist of subchains of two different type of monomers, say $A$ - and $B$-monomers. The different type of subchains tend to segregate, and hence the phase separation take place. However since the subchains are chemically bonded, the two subchains mix on a macroscopic scale, while on a molecular scale, $A$ - and $B$-subchains still segregate and the micro-domains are formed. This is called micro phase separation. For more physical background on this phenomenon we refer to $[2,8]$.

In the strong segregation regime, energetically favorable configurations have been characterized in the Ohta-Kawasaki theory [18] by minimizers of an energy functional, which is in the two dimensional case of the form

Communicated by J. Ball.

$凶$ Barbara Niethammer

niethammer@iam.uni-bonn.de

Yoshihito Oshita

oshita@okayama-u.ac.jp

1 Institute for Applied Mathematics, University of Bonn, Endenicher Allee 60, 53115 Bonn, Germany

2 Department of Mathematics, Okayama University, Tsushimanaka 3-1-1, Okayama 700-8530, Japan 


$$
E(\Omega)=\mathcal{H}^{1}(\partial \Omega)+\frac{\sigma}{2} \int_{(0, L)^{2}}\left|(-\Delta)^{-1 / 2}(\chi-\rho)\right|^{2} d x .
$$

Here $(0, L)^{2} \subset \mathbb{R}^{2}$ is the domain covered by the copolymers, $\Omega \subset[0, L)^{2}$ denotes the region covered by, say, $A$-monomers, $\rho=\frac{|\Omega|}{L^{2}} \in(0,1)$ the average density, $\sigma \in \mathbb{R}_{+}=(0, \infty)$ is a parameter related to the polymerization index, $\chi$ is the characteristic function of $\Omega$, and $\mathcal{H}^{1}$ denotes one dimensional Hausdorff measure.

The first term in the energy prefers large blocks of monomers, the second favors a very fine mixture. Competition between these terms leads to minimizers of $E$ which represent micro phase separation.

Starting with the pioneering work [15], where the Ohta-Kawasaki theory is formulated on a bounded domain as a singularly perturbed problem and the limiting sharp interface problem is identified, there has been a large body of analytical work. Minimizers of the energy functionals have been characterized in $[1,3,4,20]$, the existence/stability of stationary solutions has been investigated in $[16,17,19,21]$ and a time dependent model has been considered in $[7,9]$. The mean field models in the three dimensional case have been derived in $[6,10,12]$.

We consider the gradient flow of the energy, which is a standard way to set up a model for the evolution of the copolymer configuration that decreases energy and preserves the volume fraction. Then the evolution equation becomes the following extension of the MullinsSekerka evolution for phase separation in binary alloys [11]. The normal velocity $v$ of the interface $\partial \Omega=\partial \Omega(t)$ satisfies

$$
v=[\nabla w \cdot \vec{n}] \quad \text { on } \partial \Omega,
$$

where $[\nabla w \cdot \vec{n}]$ denotes the jump of the normal component of the gradient of the potential across the interface. Here $\vec{n}$ denotes the outer normal to $\Omega$ and

$$
[f]=\lim _{\substack{x \notin \Omega \\ x \rightarrow \partial \Omega}} f(x)-\lim _{\substack{x \in \Omega \\ x \rightarrow \partial \Omega}} f(x) .
$$

The potential $w$ is for each time determined via

$$
\begin{aligned}
-\Delta w & =0 & & \text { in }(0, L)^{2} \backslash \partial \Omega, \\
w & =\kappa+\sigma(-\Delta)^{-1}(\chi-\rho) & & \text { on } \partial \Omega,
\end{aligned}
$$

where $\kappa$ is the curvature of $\partial \Omega$. We are interested in the case that the volume of $\Omega(t)$ is preserved in time and can thus impose Neumann or periodic boundary conditions for $w$ on $\partial(0, L)^{2}$. In what follows we will consider a periodic setting and hence always require that the potential $w$ is $(0, L)^{2}$-periodic. For local well-posedness of this evolution see [5].

The evolution defined by (2)-(4) has a formal interpretation as a gradient flow of the energy (1) on a Riemannian manifold. Indeed, consider the manifold of subsets of the 2dimensional flat torus $\mathbb{T}$ of length $L$ with fixed volume, that is $\mathcal{M}:=\left\{\Omega \subset \mathbb{T} ;|\Omega|=L^{2} \rho\right\}$, whose tangent space $T_{\Omega} \mathcal{M}$ at an element $\Omega \in \mathcal{M}$ is described by all kinematically admissible normal velocities of $\partial \Omega$, that is,

$$
T_{\Omega} \mathcal{M}=\left\{v: \partial \Omega \rightarrow \mathbb{R} ; \int_{\partial \Omega} v d S=0\right\} .
$$

The Riemannian structure is given by the following metric tensor on the tangent space:

$$
g_{\Omega}\left(v^{1}, v^{2}\right)=\int_{\mathbb{T}} \nabla w^{1} \cdot \nabla w^{2} d x
$$


where $w^{\alpha}: \mathbb{T} \rightarrow \mathbb{R}(\alpha=1,2)$ solves

$$
\begin{aligned}
-\Delta w^{\alpha} & =0 & & \text { in } \mathbb{T} \backslash \partial \Omega, \\
{\left[\nabla w^{\alpha} \cdot \vec{n}\right] } & =v^{\alpha} & & \text { on } \partial \Omega
\end{aligned}
$$

for $v^{\alpha} \in T_{\Omega} \mathcal{M}(\alpha=1,2)$. The gradient flow of the energy (1) is now the dynamical system where at each time the velocity is the element of the tangent space in the direction of steepest descent of the energy. In other words, $v$ is such that

$$
g_{\Omega(t)}(v, \tilde{v})=-\langle D E(\Omega(t)), \tilde{v}\rangle
$$

for all $\tilde{v} \in T_{\Omega(t)} \mathcal{M}$. Choosing $\tilde{v}=v$ we immediately obtain the energy estimate associated with each gradient flow, which is

$$
\int_{0}^{T} g_{\Omega(t)}(v, v) d t+E(\Omega(T))=E(\Omega(0)) \text { for all } T>0 .
$$

In what follows we consider the micro phase separation in the two dimensional case in the regime where the fraction of $A$-monomers is much smaller than the one of $B$-monomers. In this case $A$-phase consists of an ensemble of many small approximately circular particles. We reduce the evolution to the gradient flow on circular particles.

For that purpose we define the submanifold $\mathcal{N} \subset \mathcal{M}$ of all sets $\Omega$ which are the union of disjoint balls $\Omega=\bigcup_{i} B_{R_{i}}\left(X_{i}\right)$, where the centers $\left\{X_{i}\right\}_{i}$ and the radii $\left\{R_{i}\right\}_{i}$ are variables. Hence $\mathcal{N}$ can be identified with an open subspace of the hypersurface $\left\{\mathbf{Y}=\left\{R_{i}, X_{i}\right\}_{i} ;\left(R_{i}, X_{i}\right) \in \mathbb{R}_{+} \times \mathbb{T}, \pi \sum_{i} R_{i}^{2}=L^{2} \rho\right\}$ in $\mathbb{R}^{3 N}$, where $N$ is the number and $i=1, \ldots, N$ an enumeration of the particles with centers in the torus $\mathbb{T}$. Since the normal velocity $v$ satisfies $v=\frac{d R_{i}}{d t}+\frac{d X_{i}}{d t} \cdot \vec{n}$ on $\partial B_{R_{i}}\left(X_{i}\right)$, the tangent space can be identified with the hyperplane

$$
T_{\mathbf{Y}} \mathcal{N}=\left\{\mathbf{Z}=\sum_{i}\left(V_{i} \frac{\partial}{\partial R_{i}}+\xi_{i} \cdot \frac{\partial}{\partial X_{i}}\right) ;\left(V_{i}, \xi_{i}\right) \in \mathbb{R} \times \mathbb{R}^{2}, \sum_{i} R_{i} V_{i}=0\right\} \subset \mathbb{R}^{3 N},
$$

such that $V_{i}$ describes the rate of change of the radius of particle $i$ and $\xi_{i}$ the rate of change of its center. We use the abbreviation $\mathbf{Z}=\left\{V_{i}, \xi_{i}\right\}_{i}$ for $\mathbf{Z}=\sum_{i}\left(V_{i} \frac{\partial}{\partial R_{i}}+\xi_{i} \cdot \frac{\partial}{\partial X_{i}}\right)$.

The metric tensor is then given by

$$
g_{\mathbf{Y}}\left(\mathbf{Z}^{1}, \mathbf{Z}^{2}\right)=\int_{\mathbb{T}} \nabla w^{1} \cdot \nabla w^{2} d x
$$

where the function $w^{\alpha}: \mathbb{T} \rightarrow \mathbb{R}$ solves

$$
\begin{aligned}
-\Delta w^{\alpha} & =0 & & \text { in } \mathbb{T} \backslash \cup_{i} \partial B_{R_{i}}\left(X_{i}\right), \\
{\left[\nabla w^{\alpha} \cdot \vec{n}\right] } & =V_{i}^{\alpha}+\xi_{i}^{\alpha} \cdot \vec{n} & & \text { on } \partial B_{R_{i}}\left(X_{i}\right) .
\end{aligned}
$$

for $\mathbf{Z}^{\alpha}=\left\{V_{i}^{\alpha}, \xi_{i}^{\alpha}\right\}_{i} \in T_{\mathbf{Y}} \mathcal{N}, \alpha=1,2$. For the following it will be convenient to split the metric tensor into the radial and shift part respectively. For any $\left\{V_{i}, \xi_{i}\right\}_{i}$, we write

$$
w=u+\phi
$$

where $u$ and $\phi$ are harmonic in- and outside the particles and where

$$
\begin{aligned}
& {[\nabla u \cdot \vec{n}]=V_{i} \quad \text { on } \partial B_{R_{i}}\left(X_{i}\right),} \\
& {[\nabla \phi \cdot \vec{n}]=\xi_{i} \cdot \vec{n} \quad \text { on } \partial B_{R_{i}}\left(X_{i}\right) \text {. }}
\end{aligned}
$$


We consider the energy $E(\mathbf{Y})=E_{\text {surf }}(\mathbf{Y})+\sigma E_{\mathrm{nl}}(\mathbf{Y})$, where

$$
E_{\text {surf }}(\mathbf{Y})=2 \pi \sum_{i} R_{i} \quad \text { and } \quad E_{\mathrm{nl}}(\mathbf{Y})=\int_{\mathbb{T}}|\nabla \mu|^{2} d x
$$

with $\mu: \mathbb{T} \rightarrow \mathbb{R}$ solving $-\Delta \mu=\chi \cup B_{R_{i}}-\rho$. We obtain the differentials of the energies in the direction of a tangent vector $\tilde{\mathbf{Z}}=\left\{\tilde{V}_{i}, \tilde{\xi}_{i}\right\}_{i}$ as

$$
\left\langle D E_{\text {surf }}(\mathbf{Y}), \tilde{\mathbf{Z}}\right\rangle=2 \pi \sum_{i} \tilde{V}_{i}
$$

and

$$
\left\langle D E_{\mathrm{nl}}(\mathbf{Y}), \tilde{\mathbf{Z}}\right\rangle=-2 \int_{\mathbb{T}} \nabla \mu \cdot \nabla \tilde{w} d x=2 \sum_{i} \int_{\partial B_{R_{i}}\left(X_{i}\right)} \mu\left(\tilde{V}_{i}+\tilde{\xi}_{i} \cdot \vec{n}\right) d S .
$$

Here $\tilde{w}: \mathbb{T} \rightarrow \mathbb{R}$ is a function $w^{\alpha}$ satisfying (9) for $\mathbf{Z}^{\alpha}=\tilde{\mathbf{Z}}$. The integration by parts yields

$$
g_{\mathbf{Y}}(\mathbf{Z}, \tilde{\mathbf{Z}})=-\sum_{i} \int_{\partial B_{R_{i}}\left(X_{i}\right)} w\left(\tilde{V}_{i}+\tilde{\xi}_{i} \cdot \vec{n}\right) d S .
$$

From now on we consider an arrangement of particles as described above which evolves according to the gradient flow equation. This means that for any $t \geq 0$, it holds for $\mathbf{Z}(t)=$ $\frac{d}{d t} \mathbf{Y}(t)$ that

$$
g_{\mathbf{Y}}(\mathbf{Z}, \tilde{\mathbf{Z}})=-\langle D E(\mathbf{Y}), \tilde{\mathbf{Z}}\rangle
$$

that is,

$$
\sum_{i} \int_{\partial B_{R_{i}}\left(X_{i}\right)} w\left(\tilde{V}_{i}+\tilde{\xi}_{i} \cdot \vec{n}\right) d S=2 \pi \sum_{i} \tilde{V}_{i}+2 \sigma \sum_{i} \int_{\partial B_{R_{i}}\left(X_{i}\right)} \mu\left(\tilde{V}_{i}+\tilde{\xi}_{i} \cdot \vec{n}\right) d S
$$

for all $\tilde{\mathbf{Z}} \in T_{\mathbf{Y}} \mathcal{N}$. Since $\tilde{\mathbf{Z}}$ is an arbitrary element of the tangent space we conclude from (11) that $w$ satisfies

$$
\frac{1}{2 \pi R_{i}} \int_{\partial B_{R_{i}}\left(X_{i}\right)}\left(w-\frac{1}{R_{i}}-2 \sigma \mu\right) d S=\lambda(t)
$$

and

$$
\int_{\partial B_{R_{i}}\left(X_{i}\right)}(w-2 \sigma \mu) \vec{n} d S=0
$$

for all $i$ such that $R_{i}>0$, with a Lagrange parameter $\lambda(t)$ that ensures volume conservation. Equations (12) and (13) are the analogue of (4) in the restricted setting.

Our aim is to identify the evolution in the limit of vanishing volume fraction of particles. More precisely, we consider a sequence of systems characterized by the parameter

$$
\varepsilon:=\left(\ln \left(\frac{d}{\mathcal{R}}\right)\right)^{-1 / 2}
$$

in the limit $\varepsilon \rightarrow 0$. Here $d$ is defined by

$$
d^{2} \sum_{i} 1=L^{2}
$$

and $\mathcal{R}$ by

$$
\mathcal{R}^{2} \sum_{i} 1=\sum_{i} R_{i}(0)^{2}
$$


Then $\frac{1}{d^{2}}$ denotes the number density of particles, and $\pi \mathcal{R}^{2}$ the average volume of particles. Here and throughout this paper we use the abbreviation $\sum_{i}=\sum_{i: R_{i}>0}$.

Our main result informally says that when $L \sim L_{s c}$, with

$$
L_{s c}^{2}:=d^{2} \ln (d / \mathcal{R}) \sim d^{2} \ln (1 / \rho),
$$

on the time scale of order $\mathcal{R}^{3} \ln (1 / \rho)$, the number density of particles with radius $r$ and center $x$, denoted by $v=v(t, r, x)$ (suitably normalized), satisfies

$$
\partial_{t} v+\partial_{r}\left(\frac{1}{r^{2}}\left\{r \psi-1-\sigma r^{3}\right\} v\right)=0,
$$

where $\psi=\psi(t, x)$ satisfies for each $t$ that

$$
-\Delta \psi+2 \pi \psi \int_{0}^{\infty} v d r=2 \pi\left(\int_{0}^{\infty} \frac{1}{r} v d r+\frac{\sigma}{L^{2}} \int_{\mathbb{T}} \int_{0}^{\infty} r^{2} v d r d y\right) \text { in } \mathbb{T}
$$

in the limit $\varepsilon \rightarrow 0$. Here $\sigma$ is also suitably normalized.

We remark that on the other hand, in the case that $L \ll L_{s c}$, that is, in the very dilute case, one obtains a homogeneous version where $\psi$ is constant in space, and is replaced by $\lambda(t)$. More precisely that the number density of particles with radius $r$, denoted by $v(t, r)$ (suitably normalized), satisfies

$$
\partial_{t} v+\partial_{r}\left(\frac{1}{r^{2}}\left(\lambda r-1-\sigma r^{3}\right) v\right)=0
$$

with

$$
\lambda(t)=\frac{\int_{0}^{\infty} \frac{1}{r} v d r+\sigma \int_{0}^{\infty} r^{2} v d r}{\int_{0}^{\infty} v d r}
$$

\section{The result}

In this section, we will introduce suitably rescaled variables, state the precise assumption on our initial particle arrangement, and present the statement of our main result.

We assume from now on that $L=L_{s c}$ for the ease of presentation, and we will rescale the spatial variables by $L_{s c}$ such that

$$
L_{s c}=L=1 \text { and hence } d=\varepsilon, \mathcal{R}=\varepsilon \exp \left(-1 / \varepsilon^{2}\right)=: \alpha_{\varepsilon} .
$$

Notice that $\rho=\pi \alpha_{\varepsilon}^{2} \varepsilon^{-2}$ and $\ln (1 / \rho) \sim \varepsilon^{-2}$. We introduce $\hat{R}_{i}, \hat{t}, \hat{V_{i}}, \hat{\xi}, \hat{w}, \hat{\sigma}$ and $\hat{\mu}$ via

$$
\begin{aligned}
R_{i}(t) & =\alpha_{\varepsilon} \hat{R}_{i}(\hat{t}), \quad t=\alpha_{\varepsilon}^{3} \ln (1 / \rho) \hat{t}, \quad w(t, x)=\alpha_{\varepsilon}^{-1} \hat{w}(\hat{t}, x), \\
V_{i}(t) & =\frac{1}{\alpha_{\varepsilon}^{2} \ln (1 / \rho)} \hat{V}_{i}(\hat{t}) \sim \frac{\varepsilon^{2}}{\alpha_{\varepsilon}^{2}} \hat{V}_{i}(\hat{t}), \quad \xi_{i}(t)=\frac{\varepsilon}{\alpha_{\varepsilon}^{2}} \hat{\xi}_{i}(\hat{t}), \\
\sigma & =\frac{1}{\alpha_{\varepsilon}^{3} \ln (1 / \rho)} \hat{\sigma} \sim \frac{\varepsilon^{2}}{\alpha_{\varepsilon}^{3}} \hat{\sigma}, \quad \mu(t, x)=\frac{\alpha_{\varepsilon}^{2}}{\varepsilon^{2}} \hat{\mu}(\hat{t}, x) .
\end{aligned}
$$

From now on we only deal with the rescaled quantities and drop the hats in the notation.

We denote the joint distribution of particle centers and radii at a given time $t$ by $v_{t}^{\varepsilon} \in\left(C_{p}^{0}\right)^{*}$, which is given by

$$
\int \zeta d \nu_{t}^{\varepsilon}=\sum_{i} \varepsilon^{2} \zeta\left(R_{i}(t), X_{i}(t)\right) \quad \text { for } \zeta \in C_{p}^{0}
$$


where $C_{p}^{0}$ stands for the space of continuous functions on $\mathbb{R}_{+} \times \mathbb{T}$ with compact support contained in $\mathbb{R}_{+} \times \mathbb{T}$. Here $\mathbb{T}$ denotes the unit flat torus, and $\mathbb{R}_{+}=(0, \infty)$. Note that since $\zeta(r, x)=0$ for $r=0$, particles which have vanished do not enter the distribution. The natural space for $v_{t}^{\varepsilon}$ and its limit $v_{t}$ is the space $\left(C_{p}^{0}\right)^{*}$ of Borel measures on $\mathbb{R}_{+} \times \mathbb{T}$.

We are now going to make the assumptions on our initial particle arrangement precise. Notice first, that in view of (15) and (16) we have

$$
\int d v_{0}^{\varepsilon}=\sum_{i} \varepsilon^{2}=1 \quad \text { and } \quad \int r^{2} d v_{0}^{\varepsilon}=\sum_{i} \varepsilon^{2} R_{i}^{2}(0)=1 .
$$

It follows immediately, that

$$
\int r d \nu_{0}^{\varepsilon}=\sum_{i} \varepsilon^{2} R_{i}(0) \leq 1,
$$

that is the surface energy of the initial particle arrangement is finite.

Furthermore it is natural to assume that initially the nonlocal energy is uniformly bounded in $\varepsilon$, that is

$$
\int_{\mathbb{T}}\left|\nabla \mu^{\varepsilon}(0, x)\right|^{2} d x \leq C,
$$

where $C$ is independent of $\varepsilon$ and where $\mu^{\varepsilon}(0, x)$ satisfies $-\Delta \mu^{\varepsilon}(0, \cdot)=\frac{\varepsilon^{2}}{\alpha_{\varepsilon}^{2}} \chi \cup_{i} B_{i}(0)-\pi$ and $\int_{\mathbb{T}} \mu^{\varepsilon} d x=0$.

We will see later [cf. (84)], that the nonlocal energy controls $\sum_{i} \varepsilon^{2} R_{i}^{4}$. Hence, finiteness of the nonlocal energy initially also implies $\sum_{i} \varepsilon^{2} R_{i}^{4}(0) \leq C$. For our analysis we need a little more than this. We need a certain tightness assumption which ensures, that not too much mass is contained in very large particles as $\varepsilon \rightarrow 0$. More precisely, we assume that

$$
\sup _{\varepsilon} \sum_{R_{i} \geq M} \varepsilon^{2} R_{i}^{4}(0) \rightarrow 0 \quad \text { as } M \rightarrow \infty
$$

Finally, we assume that initially particles are well separated in the sense that we assume that there is $\gamma>0$, such that

$$
\left\{B_{2 \gamma \varepsilon}\left(X_{i}(0)\right)\right\}_{i} \quad \text { are disjoint. }
$$

In accordance with the notation in (22) we will use in the following the abbreviation $\int \zeta d v_{t}:=\int_{0}^{\infty} \int_{\mathbb{T}} \zeta(r, x) d v_{t}(r, x)$ for $v_{t} \in\left(C_{p}^{0}\right)^{*}$. Otherwise the domain of integration is specified.

The natural space for potentials of diffusion fields is $H^{1}(\mathbb{T})$. Furthermore we will denote by $\stackrel{\circ}{H}^{1}(\mathbb{T})$ the subspace of $H^{1}(\mathbb{T})$ of functions with mean value zero.

We can now state our main result which informally says that $v_{t}^{\varepsilon}$ converges as $\varepsilon \rightarrow 0$ to a weak solution of (18)-(19).

Theorem 2.1 Let $T>0$ be given and assume that the assumptions in Sect. 2 are satisfied. Then there exists a subsequence, again denoted by $\varepsilon \rightarrow 0$, and a weakly continuous map $[0, T] \ni t \mapsto v_{t} \in\left(C_{p}^{0}\right)^{*}$ with

$$
\int \zeta d v_{t}^{\varepsilon} \rightarrow \int \zeta d v_{t} \quad \text { uniformly in } t \in[0, T] \text { for all } \zeta \in C_{p}^{0} \text {, }
$$


$\int r^{2} d v_{t}=1$ for all $t \in[0, T]$. Furthermore, there exists a measurable map $(0, T) \ni t \mapsto$ $\psi(t) \in H^{1}(\mathbb{T})$ such that (18) and (19) hold in the following weak sense

$$
\frac{d}{d t} \int \zeta d v_{t}=\int \partial_{r} \zeta \frac{1}{r^{2}}\left(r \psi(t, x)-1-\sigma r^{3}\right) d v_{t}
$$

distributionally on $(0, T)$ for all $\zeta \in C_{p}^{0}$ with $\partial_{r} \zeta \in C_{p}^{0}$. Here

$$
\int_{\mathbb{T}} \nabla \psi(t, x) \cdot \nabla \zeta-2 \pi \sigma \zeta d x+2 \pi \int \zeta\left(\psi(t, x)-\frac{1}{r}\right) d \nu_{t}=0
$$

for all $\zeta \in H^{1}(\mathbb{T})$ and almost all $t \in(0, T)$.

The proof of Theorem 2.1 goes similarly to the approach for the three dimensional case in [12]. However in contrast to the three dimensional case we need to estimate $1 / R_{i}$ term in the proof that the tightness property is preserved in time (see Lemmas 3.9 and 3.10) since the Lagrange multiplier diverges when particles disappear.

\section{Proof of Theorem 2.1}

We can deduce Theorem 2.1 by the homogenization of Rayleigh Principle (see Theorem 3.11). This will be obtained from the homogenization of metric tensor (Lemmas 3.5, 3.6) and the limit of the differential of the energy (Lemma 3.8). Also we need some a-priori estimates, which are given by a series of lemmas. The proof of Lemmas in this section will be given in Sect. 4. For readers convenience we will not abbreviate the arguments.

\subsection{Gradient flow structure}

In rescaled variables the submanifold $\mathcal{N}$ turns into

$$
\mathcal{N}^{\varepsilon}=\left\{\mathbf{Y}^{\varepsilon}=\left\{R_{i}, X_{i}\right\}_{i} ; \sum_{i} \varepsilon^{2} R_{i}^{2}=1\right\}
$$

and the tangent space

$$
T_{\mathbf{Y}^{\varepsilon}} \mathcal{N}^{\varepsilon}=\left\{\tilde{\mathbf{Z}}^{\varepsilon}=\sum_{i}\left(\tilde{V}_{i} \frac{\partial}{\partial R_{i}}+\varepsilon \alpha_{\varepsilon} \ln (1 / \rho) \tilde{\xi}_{i} \cdot \frac{\partial}{\partial X_{i}}\right) ; \sum_{i} R_{i} \tilde{V}_{i}=0\right\} .
$$

We use the abbreviation $\tilde{\mathbf{Z}}^{\varepsilon}=\left\{\tilde{V}_{i}, \tilde{\xi}_{i}\right\}_{i}$ for $\tilde{\mathbf{Z}}^{\varepsilon}=\sum_{i}\left(\tilde{V}_{i} \frac{\partial}{\partial R_{i}}+\varepsilon \alpha_{\varepsilon} \ln (1 / \rho) \tilde{\xi}_{i} \cdot \frac{\partial}{\partial X_{i}}\right)$, and $\operatorname{regard}\left\{\tilde{V}_{i}, \tilde{\xi}_{i}\right\}_{i}$ as the component of a tangent vector $\tilde{\mathbf{Z}}^{\varepsilon}$ with respect to a basis

$$
\left\{\frac{\partial}{\partial R_{i}}, \varepsilon \alpha_{\varepsilon} \ln (1 / \rho) \frac{\partial}{\partial X_{i}}\right\}_{i} \text {. }
$$

We will always denote by $\mathbf{Z}^{\varepsilon}=\left\{V_{i}, \xi_{i}\right\}_{i}$ the direction of steepest descent. Recall that $V_{i}=\frac{d R_{i}}{d t}$, but $\xi_{i}=\left(\varepsilon \alpha_{\varepsilon} \ln (1 / \rho)\right)^{-1} \frac{d X_{i}}{d t}$. The notation $\tilde{\mathbf{Z}}^{\varepsilon}$ will be used for an arbitrary element of the tangent space. Furthermore we use the abbreviation $B_{i}:=B_{\alpha_{\varepsilon} R_{i}}\left(X_{i}\right)$.

We define the energy in rescaled variables as

$$
\begin{aligned}
& E_{\varepsilon}\left(\mathbf{Y}^{\varepsilon}\right)=E_{\mathrm{surf}, \varepsilon}\left(\mathbf{Y}^{\varepsilon}\right)+\sigma E_{\mathrm{nl}, \varepsilon}\left(\mathbf{Y}^{\varepsilon}\right) \\
& E_{\mathrm{surf}, \varepsilon}\left(\mathbf{Y}^{\varepsilon}\right)=2 \pi \sum_{i} \varepsilon^{2} R_{i}, \quad E_{\mathrm{nl}, \varepsilon}\left(\mathbf{Y}^{\varepsilon}\right)=\int_{\mathbb{T}}\left|\nabla \mu^{\varepsilon}\right|^{2} d x,
\end{aligned}
$$


where $\mu^{\varepsilon}=\mu^{\varepsilon}(t, x)$ solves $-\Delta \mu^{\varepsilon}=\frac{\varepsilon^{2}}{\alpha_{\varepsilon}^{2}} \chi \cup B_{i}-\pi$ and $\int_{\mathbb{T}} \mu^{\varepsilon} d x=0$, and the metric tensor for $\tilde{\mathbf{Z}}^{\varepsilon} \in T_{\mathbf{Y}^{\varepsilon}} \mathcal{N}^{\varepsilon}$ is computed via

$$
g_{\mathbf{Y}^{\varepsilon}}\left(\tilde{\mathbf{Z}}^{\varepsilon}, \tilde{\mathbf{Z}}^{\varepsilon}\right)=\int_{\mathbb{T}}\left|\nabla \tilde{w}^{\varepsilon}\right|^{2} d x=\int_{\mathbb{T}}\left|\nabla \tilde{u}^{\varepsilon}+\nabla \tilde{\phi}^{\varepsilon}\right|^{2} d x,
$$

where $\tilde{w}^{\varepsilon}=\tilde{u}^{\varepsilon}+\tilde{\phi}^{\varepsilon}$,

$$
\begin{aligned}
& \int_{\mathbb{T}} \nabla \tilde{u}^{\varepsilon} \cdot \nabla \zeta d x+\sum_{i} \int_{\partial B_{i}} \frac{\varepsilon^{2}}{\alpha_{\varepsilon}} \tilde{V}_{i} \zeta d S=0, \\
& \int_{\mathbb{T}} \nabla \tilde{\phi}^{\varepsilon} \cdot \nabla \zeta d x+\sum_{i} \int_{\partial B_{i}} \frac{\varepsilon}{\alpha_{\varepsilon}} \tilde{\xi}_{i} \cdot \vec{n} \zeta d S=0
\end{aligned}
$$

for all $\zeta \in \stackrel{\circ}{H}^{1}(\mathbb{T})$. Notice that the potentials are only determined up to additive constants. In what follows we fix this constant by requiring that $\int_{\mathbb{T}} \tilde{u}^{\varepsilon} d x=\int_{\mathbb{T}} \tilde{\phi}^{\varepsilon} d x=0$. For the steepest descent directions $\mathbf{Z}^{\varepsilon}=\left\{V_{i}, \xi_{i}\right\}_{i}$, we define the potentials $w^{\varepsilon}, u^{\varepsilon}, \phi^{\varepsilon}$ analogously.

Equations (12) and (13) for the direction of steepest descent, turn into

$$
\frac{1}{\left|\partial B_{i}\right|} \int_{\partial B_{i}}\left(u^{\varepsilon}+\phi^{\varepsilon}-2 \sigma \mu^{\varepsilon}\right) d S=\frac{1}{R_{i}}+\lambda^{\varepsilon}(t)
$$

for some $\lambda^{\varepsilon}(t) \in \mathbb{R}$ and

$$
\int_{\partial B_{i}}\left(u^{\varepsilon}+\phi^{\varepsilon}-2 \sigma \mu^{\varepsilon}\right) \vec{n} d S=0
$$

for all $i$ such that $R_{i}>0$. Here and in what follows we abbreviate, with some abuse of notations, for a disk $B_{R}(X)$ the perimeter by $\left|\partial B_{R}\right|$ and its area by $\left|B_{R}\right|$. Now the energy estimate (8) reads

$$
\begin{aligned}
& \int_{0}^{t_{1}} \int_{\mathbb{T}}\left|\nabla w^{\varepsilon}\right|^{2} d x d t+4 \pi \sum_{i} \varepsilon^{2} \frac{R_{i}^{2}}{2}\left(t_{1}\right)+\sigma \int_{\mathbb{T}}\left|\nabla \mu^{\varepsilon}\left(t_{1}\right)\right|^{2} d x \\
& \quad=4 \pi \sum_{i} \varepsilon^{2} \frac{R_{i}^{2}}{2}(0)+\sigma \int_{\mathbb{T}}\left|\nabla \mu^{\varepsilon}(0)\right|^{2} d x
\end{aligned}
$$

for all $t_{1}>0$. Finally, the Rayleigh principle says that $\mathbf{Z}^{\varepsilon}$ satisfies

$$
\begin{aligned}
& \int_{0}^{T} \beta(t)\left(\frac{1}{2} g_{\mathbf{Y}^{\varepsilon}(t)}\left(\mathbf{Z}^{\varepsilon}, \mathbf{Z}^{\varepsilon}\right)+\left\langle D E_{\varepsilon}\left(\mathbf{Y}^{\varepsilon}(t)\right), \mathbf{Z}^{\varepsilon}\right\rangle\right) d t \\
& \quad \leq \int_{0}^{T} \beta(t)\left(\frac{1}{2} g_{\mathbf{Y}^{\varepsilon}(t)}\left(\tilde{\mathbf{Z}}^{\varepsilon}, \tilde{\mathbf{Z}}^{\varepsilon}\right)+\left\langle D E_{\varepsilon}\left(\mathbf{Y}^{\varepsilon}(t)\right), \tilde{\mathbf{Z}}^{\varepsilon}\right\rangle\right) d t
\end{aligned}
$$

for all $\tilde{\mathbf{Z}}^{\varepsilon} \in T_{\mathbf{Y}} \mathcal{N}$ and nonnegative $\beta \in C^{\infty}([0, T])$. 


\subsection{A priori estimates and weak limits}

It follows from definitions (15), (16) and the facts that volume of particles is conserved and the number can only decrease, that

$$
\begin{gathered}
\int d v_{t}^{\varepsilon} \leq 1 \text { for all } t \in[0, T], \\
\int r^{2} d v_{t}^{\varepsilon}=1 \text { for all } t \in[0, T] .
\end{gathered}
$$

On the other hand the uniform bound on the energy in (34) implies the following.

\section{Lemma 3.1}

$$
\int r^{4} d v_{t}^{\varepsilon} \leq C \quad \text { for all } t \in[0, T]
$$

Next to $v_{t}^{\varepsilon}$, we introduce signed Borel measures $\rho_{t}^{\varepsilon} \in\left(C_{p}^{0}\right)^{*}$ and $\psi_{t}^{\varepsilon} \in\left(\left(C_{p}^{0}\right)^{2}\right)^{*}$ on $\mathbb{R}_{+} \times \mathbb{T}$ via

$$
\begin{gathered}
\int \zeta d \rho_{t}^{\varepsilon}=\varepsilon^{2} \sum_{i} \zeta\left(R_{i}(t), X_{i}(t)\right) V_{i}(t) \text { for } \zeta \in C_{p}^{0}, \\
\int \eta \cdot d \psi_{t}^{\varepsilon}=\varepsilon^{2} \sum_{i} \eta\left(R_{i}(t), X_{i}(t)\right) \cdot \xi_{i}(t) \text { for } \eta \in\left(C_{p}^{0}\right)^{2}
\end{gathered}
$$

The measures satisfies $\partial_{t} \nu^{\varepsilon}+\partial_{r} \rho^{\varepsilon}+\varepsilon \alpha_{\varepsilon} \ln (1 / \rho) \operatorname{div} \psi^{\varepsilon}=0$ in the sense of distributions, that is

$$
\int_{0}^{T}\left(\partial_{t} \beta(t) \int \zeta d \nu_{t}^{\varepsilon}+\beta(t)\left\{\int \partial_{r} \zeta d \rho_{t}^{\varepsilon}+\varepsilon \alpha_{\varepsilon} \ln (1 / \rho) \int \nabla_{x} \zeta \cdot d \psi_{t}^{\varepsilon}\right\}\right) d t=0
$$

for all $\zeta \in C_{p}^{0} \cap C^{\infty}$ and $\beta \in C_{0}^{\infty}([0, T])$. As will be shown in Sect. 4, we have

$$
\mathcal{D}^{\varepsilon}:=\int_{0}^{T} 2 \pi \sum_{i} \varepsilon^{2} R_{i}^{2}\left(V_{i}^{2}+\frac{1}{4}\left|\xi_{i}\right|^{2}\right) d t \leq(1+o(1)) \int_{0}^{T} \int_{\mathbb{T}}\left|\nabla w^{\varepsilon}\right|^{2} d x d t \leq C,
$$

which yields in particular

$$
\mathcal{D}:=\liminf _{\varepsilon \rightarrow 0} \mathcal{D}^{\varepsilon} \leq \limsup _{\varepsilon \rightarrow 0} \mathcal{D}^{\varepsilon}<\infty .
$$

Bounds (39) and (40) yield a weak Hölder regularity in $t$ of $\left\{v_{t}^{\varepsilon}\right\}_{t}$ :

$$
\begin{aligned}
& \left|\int \zeta d v_{t_{1}}^{\varepsilon}-\int \zeta d v_{t_{1}}^{\varepsilon}\right| \leq\left|t_{1}-t_{2}\right|^{1 / 2} \\
& \quad\left(\frac{\mathcal{D}^{\varepsilon}}{2 \pi} \sup _{t} \int\left(\left|\partial_{r} \zeta\right|^{2}+4 \varepsilon^{2} \alpha_{\varepsilon}^{2}\left(\ln \frac{1}{\rho}\right)^{2}\left|\nabla_{x} \zeta\right|^{2}\right) \frac{1}{r^{2}} d v_{t}^{\varepsilon}\right)^{1 / 2} .
\end{aligned}
$$

Using Arzela-Ascoli's Theorem, (36) and (42) imply that there exists a weakly continuous family $\left\{v_{t}\right\}_{t}$ of nonnegative Borel measures on $\mathbb{R}_{+} \times \mathbb{T}$ such that for a subsequence

$$
\int \zeta d v_{t}^{\varepsilon} \rightarrow \int \zeta d v_{t} \text { uniformly in } t \in[0, T]
$$


for $\zeta$ in a countable subset of $C_{p}^{0} \cap C^{\infty}$. Again by (36), we see that we can extend the locally uniform convergence in (43) to all $\zeta \in C_{p}^{0}$. Obviously, the bound (36) is conserved

$$
\int d v_{t} \leq 1 \text { for all } t \in[0, T]
$$

and due to (37) and (38) we have

$$
\begin{aligned}
& \int r^{2} d v_{t}=1 \text { for all } t \in[0, T], \\
& \int r^{4} d v_{t} \leq C \text { for all } t \in[0, T] .
\end{aligned}
$$

The uniform control of the signed Borel measures $\left\{r^{2} d \rho_{t}^{\varepsilon} d t\right\}_{\varepsilon}$ and $\left\{r^{2} d \psi_{t}^{\varepsilon} d t\right\}_{\varepsilon}$ on $\mathbb{R}_{+} \times$ $\mathbb{T} \times[0, T]$ implied by (40) ensures the weak convergence, where the limits can be regarded as bounded linear functionals on $L^{2}\left(r^{2} d v_{t} d t\right)$ and $L^{2}\left(r^{2} d v_{t} d t\right)^{2}$ respectively, and hence by Riesz Representation Theorem, there exist $v \in L^{2}\left(r^{2} d v_{t} d t\right)$ and $\xi \in L^{2}\left(r^{2} d v_{t} d t\right)^{2}$ with

$$
\int_{0}^{T} \int\left(|v(t)|^{2}+|\xi(t)|^{2}\right) r^{2} d v_{t} d t \leq \frac{\mathcal{D}}{2 \pi},
$$

such that for a subsequence

$$
\begin{aligned}
\int_{0}^{T} \beta(t) \int \zeta d \rho_{t}^{\varepsilon} d t & \rightarrow \int_{0}^{T} \beta(t) \int \zeta v(t) d v_{t} d t, \\
\int_{0}^{T} \beta(t) \int \eta \cdot d \psi_{t}^{\varepsilon} d t & \rightarrow \int_{0}^{T} \beta(t) \int \eta \cdot \xi(t) d v_{t} d t,
\end{aligned}
$$

for all $\beta \in C^{0}([0, T]), \zeta \in C_{p}^{0}$ and $\eta \in\left(C_{p}^{0}\right)^{2}$, and

$$
\int_{0}^{T} \beta \int|\xi(t)|^{2} r^{2} d v_{t} d t \leq \liminf _{\varepsilon \rightarrow 0} \int_{0}^{T} \beta \sum_{i} \varepsilon^{2} R_{i}^{2}\left|\xi_{i}\right|^{2} d t
$$

for all nonnegative $\beta \in C^{0}([0, T])$. Thus the limit of (39) is

$$
\int_{0}^{T}\left(\partial_{t} \beta(t) \int \zeta d v_{t}+\beta(t) \int \partial_{r} \zeta v(t) d v_{t}\right) d t=0
$$

for all $\zeta \in C_{p}^{0} \cap C^{\infty}$ and $\beta \in C_{0}^{\infty}([0, T])$.

Definition 3.2 For $\tilde{\mathbf{Z}}^{\varepsilon}$ satisfying $\int_{0}^{T} g_{\mathbf{Y}^{\varepsilon}}\left(\tilde{\mathbf{Z}}^{\varepsilon}, \tilde{\mathbf{Z}}^{\varepsilon}\right) d t \leq C$, we can define $\tilde{v} \in L^{2}\left(r^{3} d v_{t} d t\right)$ and $\tilde{\xi} \in L^{2}\left(r^{3} d v_{t} d t\right)^{2}$ analogously. We say that $\tilde{\mathbf{Z}}^{\varepsilon}$ converges weakly to $(\tilde{v}, \tilde{\xi})$.

We are going to show below that for any $T>0$, particles do not collide on a time interval $[0, T]$ for sufficiently small $\varepsilon$. More precisely

Lemma 3.3 For any $T>0$ we can find $\varepsilon_{0}>0$ such that

$$
\left\{B_{\gamma \varepsilon}\left(X_{i}(t)\right)\right\}_{i} \quad \text { are disjoint for all } t \in[0, T],
$$

for all $\varepsilon \in\left(0, \varepsilon_{0}\right]$. 
Thus it follows that the marginal of $v_{t}$ with respect to $x$ has a bounded Lebesgue density. Hence it follows from (46) that the functional

$$
\langle L(t), \zeta\rangle:=\int \zeta r^{2} d v_{t}-\int_{\mathbb{T}} \zeta d x, \quad \zeta \in H^{1}(\mathbb{T})
$$

is an element of $H^{-1}(\mathbb{T})=\left(H^{1}(\mathbb{T})\right)^{*}$ for all $t \in[0, T]$. Hence $K(t, \cdot) \in H^{1}(\mathbb{T})$ is uniquely determined via (29) up to additive constants.

In order to prove Lemma 3.3 we show the following.

Lemma 3.4 (slow motion of the particle centers) As long as (51) is satisfied, we have

$$
\left|\xi_{i}(t)\right| \leq C \frac{\alpha_{\varepsilon}}{\varepsilon^{2}}\left(\int_{\mathbb{T}}\left|\nabla \phi^{\varepsilon}\right|^{2}+\left|\nabla u^{\varepsilon}\right|^{2}+\left|\nabla \mu^{\varepsilon}\right|^{2} d x\right)^{1 / 2} .
$$

\subsection{Homogenization of the metric tensor}

We identify the $\Gamma$-limit for the metric tensor and provide the necessary results to pass to the limit in the metric tensor. The following is a lower semicontinuity result.

Lemma 3.5 (lower semicontinuity) For all nonnegative $\beta=\beta(t) \in C^{\infty}([0, T])$ we have

$$
\begin{aligned}
& \liminf _{\varepsilon \rightarrow 0} \int_{0}^{T} \beta g_{\mathbf{Y}^{\varepsilon}}\left(\mathbf{Z}^{\varepsilon}, \mathbf{Z}^{\varepsilon}\right) d t=\liminf _{\varepsilon \rightarrow 0} \int_{0}^{T} \beta \int_{\mathbb{T}}\left|\nabla w^{\varepsilon}\right|^{2} d x d t \\
& \quad=\liminf _{\varepsilon \rightarrow 0} \int_{0}^{T} \beta \int_{\mathbb{T}}\left|\nabla u^{\varepsilon}\right|^{2}+2 \nabla u^{\varepsilon} \cdot \nabla \phi^{\varepsilon}+\left|\nabla \phi^{\varepsilon}\right|^{2} d x d t \\
& \geq \int_{0}^{T} \beta \int_{\mathbb{T}}|\nabla u|^{2} d x d t+2 \pi \int_{0}^{T} \beta \int\left(|v|^{2}+\frac{1}{4}|\xi|^{2}\right) r^{2} d v_{t} d t,
\end{aligned}
$$

where for almost all the function $u(t, \cdot) \in \stackrel{\circ}{H}^{1}(\mathbb{T})$ is determined via

$$
\int_{\mathbb{T}} \nabla u(t) \cdot \nabla \zeta d x+2 \pi \int \zeta r v(t) d v_{t}=0
$$

for all $\zeta \in \stackrel{\circ}{H}^{1}(\mathbb{T})$.

Furthermore we show that for any tangent vector of the limit manifold $(\tilde{v}, \tilde{\xi})$, there exists an approximating sequence along which the metric tensor is continuous.

Lemma 3.6 (construction) For any $\tilde{v} \in L^{2}\left(r^{2} d v_{t} d t\right)$ with $\int \tilde{v} r^{2} d v_{t}=0$ for almost all $t$ and any $\tilde{\xi} \in L^{2}\left(r^{2} d v_{t} d t\right)^{3}$ there exists a sequence $\tilde{\mathbf{Z}}^{\varepsilon}$ with $\tilde{\mathbf{Z}}^{\varepsilon} \in T_{\mathbf{Y}^{\varepsilon}} \mathcal{N}^{\varepsilon}$ such that $\tilde{\mathbf{Z}}^{\varepsilon}$ weakly converges to $(\tilde{v}, \tilde{\xi})$, and

$$
\begin{aligned}
& \limsup _{\varepsilon \rightarrow 0} \int_{0}^{T} \beta g_{\mathbf{Y}^{\varepsilon}}\left(\tilde{\mathbf{Z}}^{\varepsilon}, \tilde{\mathbf{Z}}^{\varepsilon}\right) d t \\
& \quad \leq \int_{0}^{T} \beta \int_{\mathbb{T}}|\nabla \tilde{u}|^{2} d x d t+2 \pi \int_{0}^{T} \beta \int\left(|\tilde{v}|^{2}+\frac{1}{4}|\tilde{\xi}|^{2}\right) r^{2} d \nu_{t} d t
\end{aligned}
$$

for all nonnegative $\beta=\beta(t) \in C^{\infty}([0, T])$ and with $\tilde{u}(t, \cdot) \in \stackrel{\circ}{H}^{1}(\mathbb{T})$ determined via

$$
\int_{\mathbb{T}} \nabla \tilde{u}(t) \cdot \nabla \zeta d x+2 \pi \int \zeta r \tilde{v}(t) d v_{t}=0
$$

for all $\zeta \in \stackrel{\circ}{H}^{1}(\mathbb{T})$. 
Note that the contribution from the drift term and the radial part do not interact in the limit $\varepsilon \rightarrow 0$.

\subsection{The limit of the differential of the energy}

We identify the limit of the differential of the energy. To that aim we identify the limit of the potentials $\mu^{\varepsilon}$ so as to prove the convergence for the nonlocal part of the energy.

Note that

$$
\left\langle D E_{\mathrm{nl}, \varepsilon}\left(\mathbf{Y}^{\varepsilon}\right), \tilde{\mathbf{Z}}^{\varepsilon}\right\rangle=-2 \int_{\mathbb{T}} \nabla \mu^{\varepsilon} \cdot \nabla \tilde{w}^{\varepsilon} d x .
$$

Here, as also in the homogenization of the metric tensor, the key idea is that the potentials can be represented as a sum of monopoles, which represent the self-interaction of particles, plus a slowly varying field, which represents the interaction between different particles.

We set $l=\gamma \varepsilon$, where $\gamma>0$ is as in (51). We write $\mu^{\varepsilon}=\sum_{i} \tilde{\mu}_{i}+\tilde{\mu}^{\varepsilon}$ with

$$
\tilde{\mu}_{i}(x)=\left\{\begin{array}{ll}
\frac{\varepsilon^{2}}{4 \alpha_{\varepsilon}^{2}}\left(\alpha_{\varepsilon}^{2} R_{i}^{2}-\left|x-X_{i}\right|^{2}\right)+\frac{\varepsilon^{2} R_{i}^{2}}{2} \ln \frac{l}{\alpha_{\varepsilon} R_{i}} & : x \in B_{i} \\
\frac{\varepsilon^{2} R_{i}^{2}}{2} \ln \frac{l}{\left|x-X_{i}\right|} & : x \in B_{l}\left(X_{i}\right) \backslash B_{i} \\
0 & : \text { elsewhere }
\end{array} .\right.
$$

Here $\tilde{\mu}_{i}$ is chosen such that $-\Delta \tilde{\mu}_{i}=\frac{\varepsilon^{2}}{\alpha_{\varepsilon}^{2}}$ in $B_{i}$ and $\Delta \tilde{\mu}_{i}=0$ in $B_{l}\left(X_{i}\right) \backslash B_{i}$. Since

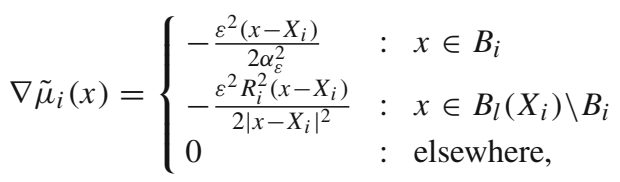

it holds that

$$
\left[\nabla \tilde{\mu}_{i} \cdot \vec{n}\right]=0 \quad \text { on } \partial B_{i} \quad \text { and } \quad\left[\nabla \tilde{\mu}_{i} \cdot \vec{n}\right]=\frac{\varepsilon^{2} R_{i}^{2}}{2 l} \quad \text { on } \partial B_{l}\left(X_{i}\right) .
$$

The slowly varying field $\tilde{\mu}^{\varepsilon}$ converges strongly to $K$ and this enables us to pass to the limit in the differential of the energy.

\section{Lemma 3.7}

$$
\sup _{t \in[0, T]}\left\|\nabla \tilde{\mu}^{\varepsilon}-\frac{1}{2} \nabla K\right\|_{L^{2}(\mathbb{T})} \rightarrow 0
$$

as $\varepsilon \rightarrow 0$. Here $K(t, \cdot) \in \stackrel{\circ}{H}^{1}(\mathbb{T})$ satisfies for each $t$

$$
\int_{\mathbb{T}} \nabla K \cdot \nabla \zeta d x=2 \pi\left(\int r^{2} \zeta d v_{t}-\int_{\mathbb{T}} \zeta d x\right)
$$

for all $\zeta \in H^{1}(\mathbb{T})$.

Lemma 3.8 Assume that $\tilde{\mathbf{Z}}^{\varepsilon}$ satisfies

$$
\int_{0}^{T} g_{\mathbf{Y}^{\varepsilon}}\left(\tilde{\mathbf{Z}}^{\varepsilon}, \tilde{\mathbf{Z}}^{\varepsilon}\right) d t \leq C
$$


and converges weakly to $(\tilde{v}, \tilde{\xi})$. Then for all $\beta \in C^{\infty}([0, T])$,

$$
\lim _{\varepsilon \rightarrow 0} \int_{0}^{T} \beta\left\langle D E_{\mathrm{nl}, \varepsilon}\left(\mathbf{Y}^{\varepsilon}\right), \tilde{\mathbf{Z}}^{\varepsilon}\right\rangle d t=\int_{0}^{T} \beta\left\langle D E_{\mathrm{nl}}\left(v_{t}\right),(\tilde{v}, \tilde{\xi})\right\rangle d t .
$$

where

$$
\left\langle D E_{\mathrm{nl}}\left(v_{t}\right),(\tilde{v}, \tilde{\xi})\right\rangle=2 \pi \int\left(r^{3}+r K(t, x)\right) \tilde{v} d v_{t}
$$

\subsubsection{Tightness}

In order to prove Lemma 3.8 we first need to show that the tightness property (26) is preserved in time so that no mass is lost at infinity in the limit $\varepsilon \rightarrow 0$.

Lemma 3.9 (tightness) For any $t>0$ we have

$$
\sum_{R_{i} \geq M} \varepsilon^{2} R_{i}^{4}(t) \rightarrow 0 \quad \text { as } M \rightarrow \infty \text { uniformly in } \varepsilon .
$$

This lemma is crucial to our proof. The proof is much more difficult than three dimensional case. In fact, the main idea of the proof is to show by asymptotics that, at least in some average sense, $V_{i}$ satisfies approximately $R_{i}^{2} V_{i} \sim u R_{i}-1-\sigma R_{i}^{3}$ where $u$ is the Lagrange multiplier that ensures the volume conservation. In contrast to the three dimensional case, the Lagrange multiplier may diverge. However we can still show the following a-priori estimate, and thus, at least on average, $V_{i} \leq 0$, if $R_{i}$ is sufficiently large, and no mass can escape to infinity as $\varepsilon \rightarrow 0$, from which one deduces Lemma 3.9.

Lemma 3.10 For any $T>0$, there exist constants $C_{T}>0$ and $\varepsilon_{1}>0$ such that

$$
\begin{aligned}
& \int_{0}^{T}\left(\int \frac{1}{1-\varepsilon^{2} \ln r} \frac{1}{r^{2}} d v_{t}^{\varepsilon}\right)^{1 / 2} d t \\
& \quad \leq C\left(\int_{0}^{T}\left(1+\int_{\mathbb{T}}\left|\nabla\left(w^{\varepsilon}-2 \sigma \mu^{\varepsilon}\right)\right|^{2} d x\right) d t\right)^{1 / 2} \leq C_{T}
\end{aligned}
$$

for all $\varepsilon \in\left(0, \varepsilon_{1}\right]$.

\subsection{Homogenization of Rayleigh principle}

The main task that remains to be done now is to determine the equation for the velocity function $v$. It will be characterized as the minimizer in the Rayleigh principle. Thus our task is to characterize the limits of $\mathbf{Z}^{\varepsilon}$ that satisfy (35).

Notice that we can use Lemma 3.8 with $\mathbf{Z}^{\varepsilon}-\tilde{\mathbf{Z}}^{\varepsilon}$ where $\mathbf{Z}^{\varepsilon}$ is the direction of steepest descent and $\tilde{\mathbf{Z}}^{\varepsilon}$ is as in Lemma 3.6. The main result from which one deduces Theorem 2.1 is the following.

Theorem 3.11 For all nonnegative $\beta \in C^{\infty}([0, T])$ we have

$$
\begin{aligned}
& \int_{0}^{T} \beta\left(\frac{1}{2} \int_{\mathbb{T}}|\nabla u|^{2} d x+2 \pi \int \frac{1}{2}\left(|v|^{2}+\frac{1}{4}|\xi|^{2}\right) r^{2} d v_{t}\right. \\
& \left.\quad+\left\langle D E\left(v_{t}\right),(v, \xi)-(\tilde{v}, \tilde{\xi})\right\rangle\right) d t \\
& \quad \leq \int_{0}^{T} \beta\left(\frac{1}{2} \int_{\mathbb{T}}|\nabla \tilde{u}|^{2} d x+2 \pi \int \frac{1}{2}\left(|\tilde{v}|^{2}+\frac{1}{4}|\tilde{\xi}|^{2}\right) r^{2} d v_{t}\right) d t
\end{aligned}
$$


for all $\tilde{v} \in L^{2}\left(r^{2} d v_{t} d t\right)$ and $\tilde{\xi} \in L^{2}\left(r^{2} d v_{t} d t\right)^{2}$ such that $\int r \tilde{v} d v_{t}=0$ for almost all t and such that $(v-\tilde{v})(t, x, \cdot)=0$ in a neighborhood of $r=0$. Here

$$
\left\langle D E\left(v_{t}\right),(\tilde{v}, \tilde{\xi})\right\rangle=2 \pi \int\left(1+\sigma\left(r^{3}+r K(t, x)\right)\right) \tilde{v} d v_{t}
$$

for $\tilde{v}$ which vanish in a neighborhood of $r=0$, and $u(t, \cdot), \tilde{u}(t, \cdot), K(t, \cdot) \in \stackrel{\circ}{H}^{1}(\mathbb{T})$ are determined for a.a. $t$ via (53), (55), and (59) respectively.

The Euler-Lagrange equation for (62) becomes $r^{2} v=r(u-\sigma K-\lambda)-1-\sigma r^{3}$ with $\lambda$ being a Lagrange multiplier that ensures the constraint $\int r v d v_{t}=0$. Setting $\psi(t, x) \equiv$ $u-\sigma K-\lambda$, we can then derive (28)-(29). The proof is basically straightforward and goes similarly to the one in Chapter 6 of [13]. We omit the details here.

Notice that in the formulation (62) we need that $v-\tilde{v}$ has compact support in the $r$-variable. This is due to the fact that we cannot guarantee that the term $\int r v d v_{t}$ which appears in the differential of the surface energy is well-defined.

\section{Proof of Lemmas}

Proof of Lemma 3.4 We set $l=\gamma \varepsilon$, where $\gamma$ is as in (51). Notice that due to (51) and $\sum_{i} \varepsilon^{2} R_{i}^{2}=1$, the balls $\left\{B_{l}\left(X_{i}\right)\right\}_{i}$ are disjoint. For given $\left\{\xi_{i}\right\}_{i}$ define

$$
\phi_{i}(x)= \begin{cases}-\frac{\varepsilon}{2}\left(\frac{1}{\alpha_{\varepsilon}}-\frac{\alpha_{\varepsilon} R_{i}^{2}}{l^{2}}\right)\left(x-X_{i}\right) \cdot \xi_{i} & : x \in B_{i} \\ -\frac{\varepsilon \alpha_{\varepsilon} R_{i}^{2}}{2}\left(\frac{1}{\left|x-X_{i}\right|^{2}}-\frac{1}{l^{2}}\right)\left(x-X_{i}\right) \cdot \xi_{i} & : x \in B_{l}\left(X_{i}\right) \backslash B_{i} \\ 0 & : \text { elsewhere. }\end{cases}
$$

For further use we collect some properties of $\phi_{i}$. It is easily checked that $\phi_{i}$ is continuous in $\mathbb{T}$, harmonic in $B_{l}\left(X_{i}\right) \backslash \partial B_{i}$ and satisfies

$$
\begin{aligned}
& {\left[\nabla \phi_{i} \cdot \vec{n}\right]=\frac{\varepsilon}{\alpha_{\varepsilon}} \xi_{i} \cdot \vec{n} \quad \text { on } \partial B_{i},} \\
& {\left[\nabla \phi_{i} \cdot \vec{n}\right]=-\frac{\varepsilon \alpha_{\varepsilon} R_{i}^{2}}{l^{2}} \xi_{i} \cdot \vec{n} \quad \text { on } \partial B_{l}\left(X_{i}\right) .}
\end{aligned}
$$

Furthermore, $\phi_{i}=0$ on $\partial B_{l}\left(X_{i}\right)$ and

$$
\phi_{i}=-\frac{\varepsilon R_{i}}{2}(1+o(1)) \xi_{i} \cdot \vec{n} \quad \text { on } \partial B_{i} .
$$

Then

$$
\begin{aligned}
\frac{1}{2}\left|\xi_{i}\right|^{2} & =\frac{1}{\left|\partial B_{i}\right|} \int_{\partial B_{i}}\left|\xi_{i} \cdot \vec{n}\right|^{2} d S \\
& =\frac{1}{\left|\partial B_{i}\right|} \int_{\partial B_{i}} \frac{\alpha_{\varepsilon}}{\varepsilon}\left[\nabla \phi^{\varepsilon} \cdot \vec{n}\right] \xi_{i} \cdot \vec{n} d S \\
& =-\frac{2 \alpha_{\varepsilon}}{\varepsilon^{2} R_{i}\left|\partial B_{i}\right|} \int_{\partial B_{i}}\left[\nabla \phi^{\varepsilon} \cdot \vec{n}\right] \phi_{i} d S(1+o(1)) .
\end{aligned}
$$


Integration by parts yields

$$
\begin{aligned}
\int_{\partial B_{i}}\left[\nabla \phi^{\varepsilon} \cdot \vec{n}\right] \phi_{i} d S & =-\int_{B_{l}\left(X_{i}\right) \backslash B_{i}} \nabla \phi^{\varepsilon} \cdot \nabla \phi_{i} d S \\
& =\int_{\partial B_{i}}\left[\nabla \phi_{i} \cdot \vec{n}\right] \phi^{\varepsilon} d S+\int_{\partial B_{l}\left(X_{i}\right)}\left[\nabla \phi_{i} \cdot \vec{n}\right] \phi^{\varepsilon} d S \\
& =\frac{\varepsilon \xi_{i}}{\alpha_{\varepsilon}} \cdot \int_{\partial B_{i}} \phi^{\varepsilon} \vec{n} d S-\frac{\varepsilon \alpha_{\varepsilon} R_{i}^{2}}{l^{2}} \int_{\partial B_{l}\left(X_{i}\right)} \phi^{\varepsilon} \xi_{i} \cdot \vec{n} d S .
\end{aligned}
$$

Together with (66), (67) and (33) we find

$$
\begin{aligned}
\left|\xi_{i}\right|^{2} \leq & C\left(\frac{1}{\varepsilon R_{i}} \xi_{i} \cdot \frac{1}{\left|\partial B_{i}\right|} \int_{\partial B_{i}} \phi^{\varepsilon} \vec{n} d S+\frac{\alpha_{\varepsilon}}{\varepsilon l^{2}} \int_{\partial B_{l}\left(X_{i}\right)} \phi^{\varepsilon} \xi_{i} \cdot \vec{n} d S\right) \\
\leq & C\left(\frac{1}{\varepsilon R_{i}\left|\partial B_{i}\right|}\left|\xi_{i} \cdot \int_{\partial B_{i}} \mu^{\varepsilon} \vec{n} d S\right|+\frac{1}{\varepsilon R_{i}\left|\partial B_{i}\right|} \mid \xi_{i}\right. \\
& \left.\cdot \int_{\partial B_{i}} u^{\varepsilon} \vec{n} d S\left|+\frac{\alpha_{\varepsilon}}{\varepsilon l^{2}}\right| \int_{\partial B_{l}\left(X_{i}\right)} \phi^{\varepsilon} \xi_{i} \cdot \vec{n} d S \mid\right) .
\end{aligned}
$$

We estimate the last term on the right hand side of (68) via

$$
\begin{aligned}
\frac{\alpha_{\varepsilon}}{\varepsilon l^{2}}\left|\int_{\partial B_{l}\left(X_{i}\right)} \phi^{\varepsilon} \xi_{i} \cdot \vec{n} d S\right| & =\frac{\alpha_{\varepsilon}}{\varepsilon l^{2}}\left|\int_{B_{l}\left(X_{i}\right)} \nabla \phi^{\varepsilon} \cdot \xi_{i} d x\right| \\
& \leq\left|\xi_{i}\right| \frac{\alpha_{\varepsilon}}{\varepsilon l^{2}} \int_{B_{l}\left(X_{i}\right)}\left|\nabla \phi^{\varepsilon}\right| d x \\
& \leq C\left|\xi_{i}\right| \frac{\alpha_{\varepsilon}}{\varepsilon l}\left(\int_{B_{l}\left(X_{i}\right)}\left|\nabla \phi^{\varepsilon}\right|^{2} d x\right)^{1 / 2} .
\end{aligned}
$$

We can write $\mu^{\varepsilon}=\sum_{i} \mu_{i}+\bar{\mu}^{\varepsilon}$ with

$$
\mu_{i}(x)= \begin{cases}\frac{\varepsilon^{2}}{4 \alpha_{\varepsilon}^{2}}\left(\alpha_{\varepsilon}^{2} R_{i}^{2}-\left|x-X_{i}\right|^{2}\right)+\frac{\varepsilon^{2} R_{i}^{2}}{2} \ln \frac{l}{\alpha_{\varepsilon} R_{i}}-\frac{\pi}{4}\left(l^{2}-\left|x-X_{i}\right|^{2}\right) & : x \in B_{i} \\ \frac{\varepsilon^{2} R_{i}^{2}}{2} \ln \frac{l}{\left|x-X_{i}\right|}-\frac{\pi}{4}\left(l^{2}-\left|x-X_{i}\right|^{2}\right) & : x \in B_{l}\left(X_{i}\right) \backslash B_{i} \\ 0 & : \text { elsewhere. }\end{cases}
$$

Notice that $\mu_{i}$ is continuous in $\mathbb{T}$, and satisfies $-\Delta \mu_{i}=\frac{\varepsilon^{2}}{\alpha_{\varepsilon}^{2}}-\pi$ in $B_{i},-\Delta \mu_{i}=-\pi$ in $B_{l}\left(X_{i}\right) \backslash \overline{B_{i}}$. These imply that $\bar{\mu}^{\varepsilon}$, and hence $\nabla \bar{\mu}^{\varepsilon}$ also are harmonic in $\cup_{i} B_{l}\left(X_{i}\right)$. Then, due to the properties of $\mu_{i}$ and the mean value theorem, we have

$$
\begin{aligned}
\int_{\partial B_{i}} \mu^{\varepsilon} \vec{n} d S & =\int_{B_{i}} \nabla \mu^{\varepsilon} d x=\int_{B_{i}} \nabla \mu_{i} d x+\int_{B_{i}} \nabla \bar{\mu}^{\varepsilon} d x \\
& =\nabla \mu_{i}\left(X_{i}\right)\left|B_{i}\right|+\int_{B_{i}} \nabla \bar{\mu}^{\varepsilon} d x=\int_{B_{i}} \nabla \bar{\mu}^{\varepsilon} d x \\
& =\frac{\left|B_{i}\right|}{\left|B_{l}\left(X_{i}\right)\right|} \int_{B_{l}\left(X_{i}\right)} \nabla \bar{\mu}^{\varepsilon} d x .
\end{aligned}
$$

But

$$
\int_{B_{l}\left(X_{i}\right)} \nabla \bar{\mu}^{\varepsilon} d x=\int_{\partial B_{l}\left(X_{i}\right)} \bar{\mu}^{\varepsilon} \vec{n} d S=\int_{\partial B_{l}\left(X_{i}\right)} \mu^{\varepsilon} \vec{n} d S=\int_{B_{l}\left(X_{i}\right)} \nabla \mu^{\varepsilon} d x,
$$


so

$$
\left|\int_{\partial B_{i}} \mu^{\varepsilon} \vec{n} d S\right| \leq C\left|B_{i}\right| l^{-1}\left(\int_{B_{l}\left(X_{i}\right)}\left|\nabla \mu^{\varepsilon}\right|^{2} d x\right)^{1 / 2} .
$$

Next we write $u^{\varepsilon}=\sum_{i} u_{i}+\bar{u}^{\varepsilon}$ where

$$
u_{i}(x)= \begin{cases}R_{i} V_{i} \varepsilon^{2} \ln \left(\frac{\alpha_{\varepsilon} R_{i}}{l}\right) \sim-R_{i} V_{i} & : x \in B_{i} \\ \varepsilon^{2} R_{i} V_{i} \ln \left(\frac{\left|x-X_{i}\right|}{l}\right) & : x \in B_{l}\left(X_{i}\right) \backslash B_{i} . \\ 0 & : \text { elsewhere }\end{cases}
$$

It is easily checked that $u_{i}$ is continuous in $\mathbb{T}$, harmonic in $B_{l}\left(X_{i}\right) \backslash \partial B_{i}$ and satisfies

$$
\left[\nabla u_{i} \cdot \vec{n}\right]=\frac{\varepsilon^{2}}{\alpha_{\varepsilon}} V_{i} \quad \text { on } \partial B_{i} .
$$

Then from a similar argument as above, we see that

$$
\left|\int_{\partial B_{i}} u^{\varepsilon} \vec{n} d S\right| \leq C\left|B_{i}\right| l^{-1}\left(\int_{B_{l}\left(X_{i}\right)}\left|\nabla u^{\varepsilon}\right|^{2} d x\right)^{1 / 2} .
$$

Thus, in summary we find

$$
\left|\xi_{i}\right| \leq C \frac{\alpha_{\varepsilon}}{\varepsilon l}\left(\int_{B_{l}\left(X_{i}\right)}\left|\nabla \mu^{\varepsilon}\right|^{2}+\left|\nabla u^{\varepsilon}\right|^{2}+\left|\nabla \phi^{\varepsilon}\right|^{2} d x\right)^{1 / 2} .
$$

This completes the proof of Lemma.

Proof of Lemma 3.3 In Sect. 3.3 we will see that $\int_{0}^{T} \int_{\mathbb{T}}\left|\nabla \phi^{\varepsilon}\right|^{2}+\left|\nabla u^{\varepsilon}\right|^{2} d x d t \leq C$ (cf. (81)). Furthermore, due to (34), we have $\int_{\mathbb{T}}\left|\nabla \mu^{\varepsilon}\right|^{2} d x \leq C$. Then the statement follows from Lemma 3.4 and

$$
\begin{aligned}
\left|X_{i}(t)-X_{j}(t)\right| & =\left|X_{i}(0)-X_{j}(0)+\frac{\alpha_{\varepsilon}}{\varepsilon^{2}} \int_{0}^{t} \xi_{i}(s)-\xi_{j}(s) d s\right| \\
& \geq\left|X_{i}(0)-X_{j}(0)\right|-\frac{\alpha_{\varepsilon}}{\varepsilon^{2}} \int_{0}^{t}\left|\xi_{i}(s)\right|+\left|\xi_{j}(s)\right| d s .
\end{aligned}
$$

Proof of Lemma 3.5 We can prove

$$
\liminf _{\varepsilon \rightarrow 0} \int_{0}^{T} \beta \int_{\mathbb{T}}\left|\nabla u^{\varepsilon}\right|^{2} d x d t \geq \int_{0}^{T} \beta \int_{\mathbb{T}}|\nabla u|^{2} d x d t+2 \pi \int_{0}^{T} \beta \int|v|^{2} r^{2} d v_{t} d t
$$

in the same way as in [13] since (51) is satisfied.

Hence, it remains to show that

$$
\liminf _{\varepsilon \rightarrow 0} \int_{0}^{T} \beta \int_{\mathbb{T}}\left|\nabla \phi^{\varepsilon}\right|^{2} d x d t \geq 2 \pi \int \beta \int \frac{1}{4}|\xi|^{2} r^{2} d v_{t} d t
$$

and

$$
\lim _{\varepsilon \rightarrow 0} \int_{0}^{T} \beta \int_{\mathbb{T}} \nabla u^{\varepsilon} \cdot \nabla \phi^{\varepsilon} d x d t=0
$$


Step 1: Monopoles. Our goal is to construct a good approximation of $\phi^{\varepsilon}$ which is based on cutting off the single monopole solutions. To that aim we define $\phi_{i}$ as in (63) with $\gamma$ is as in (51) and thus the balls $\left\{B_{l}\left(X_{i}\right)\right\}_{i}$ are disjoint. Using (64) and (65), we find

$$
\begin{aligned}
\int_{B_{l}\left(X_{i}\right)}\left|\nabla \phi_{i}\right|^{2} d x & =-\int_{\partial B_{i}} \phi_{i} \frac{\varepsilon}{\alpha_{\varepsilon}} \xi_{i} \cdot \vec{n} d S \\
& =\frac{1}{2}(1+o(1)) \frac{\varepsilon^{2} R_{i}}{\alpha_{\varepsilon}} \int_{\partial B_{i}}\left|\xi_{i} \cdot \vec{n}\right|^{2} d S \\
& =\frac{1}{2}(1+o(1)) 2 \pi \varepsilon^{2} R_{i}^{2} \int_{\partial B_{i}}\left|\xi_{i} \cdot \vec{n}\right|^{2} d S \\
& =\frac{\pi}{2} \varepsilon^{2} R_{i}^{2}\left|\xi_{i}\right|^{2}(1+o(1)) .
\end{aligned}
$$

Step 2: A lower bound. We will show that for any given $\delta>0$ we have

$$
\int_{\mathbb{T}}\left|\nabla \phi^{\varepsilon}\right|^{2} d x \geq(1-\delta) \frac{\pi}{2} \sum_{i} \varepsilon^{2} R_{i}^{2}\left|\xi_{i}\right|^{2}
$$

if $\varepsilon$ is sufficiently small. Indeed, due to the fact that $\left\{B_{l}\left(X_{i}\right)\right\}_{i}$ are disjoint we have

$$
\int_{\mathbb{T}}\left|\nabla \phi^{\varepsilon}\right|^{2} d x \geq \sum_{i} \int_{B_{l}\left(X_{i}\right)}\left|\nabla \phi^{\varepsilon}\right|^{2} d x .
$$

On the other hand

$$
\int_{B_{l}\left(X_{i}\right)}\left|\nabla \phi^{\varepsilon}\right|^{2} d x \geq \inf _{\psi} \int_{B_{l}\left(X_{i}\right)}|\psi|^{2} d x,
$$

where

$$
\int_{B_{l}\left(X_{i}\right)} \psi \cdot \nabla \zeta+\int_{\partial B_{i}} \frac{\varepsilon}{\alpha_{\varepsilon}} \xi_{i} \cdot \vec{n} \zeta d S=0
$$

for all $\zeta \in C_{0}^{\infty}\left(B_{l}\left(X_{i}\right)\right)$. From the corresponding Euler-Lagrange equation we see that the minimizer $\hat{\psi}$ is orthogonal to all divergence-free function and hence a gradient. We find that $\hat{\psi}=\nabla \phi_{i}$ and thus (77) follows from (76).

Step 3: Approximation of $\phi^{\varepsilon}$. With

$$
\hat{\phi}^{\varepsilon}(x):=\sum_{i} \phi_{i}(x),
$$

we have

$$
\int_{\mathbb{T}}\left|\nabla \phi^{\varepsilon}-\nabla \hat{\phi}^{\varepsilon}\right|^{2} d x \leq C \varepsilon^{k} \sum_{i} \varepsilon^{2} R_{i}^{2}\left|\xi_{i}\right|^{2}
$$

for any $k>0$. In fact, for $\bar{\phi}^{\varepsilon}:=\phi^{\varepsilon}-\hat{\phi}^{\varepsilon}$ it follows from the definitions and (64) that

$$
\int_{\mathbb{T}} \nabla \bar{\phi}^{\varepsilon} \cdot \nabla \zeta d x= \pm \sum_{i} \frac{\alpha_{\varepsilon} R_{i}^{2}}{l^{2}} \int_{\partial B_{l}\left(X_{i}\right)} \xi_{i} \cdot \vec{n} \zeta d S
$$

for all $\zeta \in \stackrel{\circ}{H}^{1}(\mathbb{T})$. We now define $L_{\varepsilon} \in\left(H^{1}(\mathbb{T})\right)^{*}$ via

$$
\left\langle L_{\varepsilon}, \zeta\right\rangle:=\sum_{i} \frac{\alpha_{\varepsilon} R_{i}^{2}}{l^{2}} \int_{\partial B_{l}\left(X_{i}\right)} \zeta \xi_{i} \cdot \vec{n} d S .
$$


We observe that due to $f_{\partial B_{l}\left(X_{i}\right)} \xi_{i} \cdot \vec{n} d S=0$ we can write

$$
\left\langle L_{\varepsilon}, \zeta\right\rangle=\sum_{i} \frac{\alpha_{\varepsilon} R_{i}^{2}}{l^{2}} \int_{\partial B_{l}\left(X_{i}\right)}\left(\zeta-f_{\partial B_{l}\left(X_{i}\right)} \zeta\right) \xi_{i} \cdot \vec{n} d S .
$$

We can then estimate

$$
\begin{aligned}
\left|\left\langle L_{\varepsilon}, \zeta\right\rangle\right| & \leq C \sum_{i} \frac{\alpha_{\varepsilon} R_{i}^{2}}{l^{2}}\left(\int_{\partial B_{l}\left(X_{i}\right)}\left|\xi_{i} \cdot \vec{n}\right|^{2} d S\right)^{1 / 2}\left(\int_{\partial B_{l}\left(X_{i}\right)}\left|\zeta-\int_{\partial B_{l}\left(X_{i}\right)} \zeta\right|^{2} d S\right)^{1 / 2} \\
& \leq C \sum_{i} \frac{\alpha_{\varepsilon} R_{i}^{2}}{l^{2}}\left(\pi l\left|\xi_{i}\right|^{2}\right)^{1 / 2}\left(C l^{2} \int_{B_{l}\left(X_{i}\right)}|\nabla \zeta|^{2} d x\right)^{1 / 2},
\end{aligned}
$$

where the last estimate follows from Trace Theorem and Poincaré's inequality. This gives

$$
\begin{aligned}
\left|\left\langle L_{\varepsilon}, \zeta\right\rangle\right| & \leq C \sum_{i} \frac{\alpha_{\varepsilon} R_{i}^{2}}{l^{2}} l^{3 / 2}\left|\xi_{i}\right|\left(\int_{B_{l}\left(X_{i}\right)}|\nabla \zeta|^{2} d x\right)^{1 / 2} \\
& \leq C\left(\sum_{i} \frac{\alpha_{\varepsilon}^{2} R_{i}^{2}}{\varepsilon^{3}} \varepsilon^{2} R_{i}^{2}\left|\xi_{i}\right|^{2}\right)^{1 / 2}\left(\sum_{i} \int_{B_{l}\left(X_{i}\right)}|\nabla \zeta|^{2} d x\right)^{1 / 2} .
\end{aligned}
$$

Due to the simple estimate $R_{i} \leq C \varepsilon^{-1 / 2}$ we find $\sup _{\zeta \neq 0} \frac{\left|\left\langle L_{\varepsilon}, \zeta\right\rangle\right|}{\|\nabla \zeta\|_{L^{2}(\mathbb{T})}} \leq C \alpha_{\varepsilon} \varepsilon^{-2}\left(\sum_{i} \varepsilon^{2} R_{i}^{2}\left|\xi_{i}\right|^{2}\right)^{1 / 2} \leq C \varepsilon^{k}\left(\sum_{i} \varepsilon^{2} R_{i}^{2}\left|\xi_{i}\right|^{2}\right)^{1 / 2} \quad$ for any $k>0$,

which proves (79).

Step 4: Bounds on the individual terms.

$$
\int_{0}^{T} \int_{\mathbb{T}}\left|\nabla u^{\varepsilon}\right|^{2}+\left|\nabla \phi^{\varepsilon}\right|^{2} d x d t \leq 2 \int_{0}^{T} \int_{\mathbb{T}}\left|\nabla w^{\varepsilon}\right|^{2} d x d t \leq C .
$$

We write

$$
\int_{\mathbb{T}} \nabla u^{\varepsilon} \cdot \nabla \phi^{\varepsilon} d x=\int_{\mathbb{T}} \nabla u^{\varepsilon} \cdot \nabla \bar{\phi}^{\varepsilon} d x+\int_{\mathbb{T}} \nabla u^{\varepsilon} \cdot \nabla \hat{\phi}^{\varepsilon} d x .
$$

Now $\nabla u^{\varepsilon}$ is orthogonal to $\nabla \hat{\phi}^{\varepsilon}$ since

$$
\int_{\mathbb{T}} \nabla u^{\varepsilon} \cdot \nabla \hat{\phi}^{\varepsilon} d x=-\sum_{i} \frac{\varepsilon^{3} V_{i}}{\alpha_{\varepsilon}} \int_{\partial B_{i}} \phi_{i} d S
$$

but $\int_{\partial B_{i}} \phi_{i} d S=0$. Thus

$$
\int_{\mathbb{T}} \nabla u^{\varepsilon} \cdot \nabla \phi^{\varepsilon} d x=\int_{\mathbb{T}} \nabla u^{\varepsilon} \cdot \nabla \bar{\phi}^{\varepsilon} d x
$$

Furthermore, for some small $\delta>0$, using (77),

$$
\begin{aligned}
\int_{\mathbb{T}} \nabla u^{\varepsilon} \cdot \nabla \bar{\phi}^{\varepsilon} d x & \geq-\delta\left\|\nabla u^{\varepsilon}\right\|_{L^{2}(\mathbb{T})}^{2}-C_{\delta}\left\|\nabla \bar{\phi}^{\varepsilon}\right\|_{L^{2}(\mathbb{T})} \\
& \geq-\delta\left\|\nabla u^{\varepsilon}\right\|_{L^{2}(\mathbb{T})}^{2}-C_{\delta} \varepsilon^{4} \sum_{i} \varepsilon^{3} R_{i}^{3}\left|\xi_{i}\right|^{2} \\
& \geq-\delta\left\|\nabla u^{\varepsilon}\right\|_{L^{2}(\mathbb{T})}^{2}-C_{\delta} \varepsilon^{4}\left\|\nabla \phi^{\varepsilon}\right\|_{L^{2}(\mathbb{T})}
\end{aligned}
$$


We choose $\delta=\frac{1}{4}$ and then $\varepsilon$ so small such that $C_{\delta} \varepsilon^{4} \leq \frac{1}{4}$. Then (81) follows from (34) and (82).

Step 5: Lower semicontinuity. Since we have now established (81), we obtain the existence of the weak limit $\xi$ as explained before in Sect. 3.2, and hence the assertion (74) follows from (49) and (77).

Step 6: The mixed term vanishes in the limit.

As an immediate consequence of (77), (79), (81) and (82) we find

$$
\int_{0}^{T}\left\|\nabla \bar{\phi}^{\varepsilon}\right\|_{L^{2}(\mathbb{T})}^{2} d t \leq C \varepsilon^{4}
$$

and thus that (75) is valid.

Proof of Lemma 3.6 We need only to show that for given $\tilde{\xi}$ as above we can find $\tilde{\phi}^{\varepsilon}$ such that

$$
\limsup _{\varepsilon \rightarrow 0} \int_{0}^{T} \beta \int_{\mathbb{T}}\left|\nabla \tilde{\phi}^{\varepsilon}\right|^{2} d x d t \leq 2 \pi \int_{0}^{T} \beta \int \frac{1}{4}|\tilde{\xi}|^{2} r^{2} d v_{t} d t
$$

and

$$
\lim _{\varepsilon \rightarrow 0} \int_{0}^{T} \beta \int_{\mathbb{T}} \nabla \tilde{u}^{\varepsilon} \cdot \nabla \tilde{\phi}^{\varepsilon} d x d t=0
$$

since the corresponding result for $\tilde{u}^{\varepsilon}$ can be proved in the same way as in [13]. The proof is in fact quite similar to the proof of Lemma 3.5, since there the minimization property of $\left\{\xi_{i}\right\}_{i}$ is not used in the construction, which is henceforth quite general.

Step 1: Construction for smooth $\tilde{\xi}$. We first assume that $\tilde{\xi}$ is smooth. Then we define $\tilde{\xi}_{i}:=$ $\tilde{\xi}\left(X_{i}\right)$ and construct $\phi_{i}$ as in (63) for $\xi_{i}=\tilde{\xi}_{i}$, and a corresponding $\hat{\phi}^{\varepsilon}$ as in (78). The property (76) implies

$$
\int_{\mathbb{T}}\left|\nabla \hat{\phi}^{\varepsilon}\right|^{2} d x=\frac{\pi}{2} \sum_{i} \varepsilon^{2}\left|\tilde{\xi}_{i}\right|^{2} R_{i}^{2}(1+o(1))
$$

and hence

$$
\begin{aligned}
\int_{0}^{T} \beta \int_{\mathbb{T}}\left|\nabla \hat{\phi}^{\varepsilon}\right|^{2} d x d t= & \pi \int_{0}^{T} \beta \int \frac{1}{2}|\tilde{\xi}|^{2} r^{2} d v_{t}^{\varepsilon} d t(1+o(1)) \\
& \rightarrow \pi \int_{0}^{T} \beta \int \frac{1}{2}|\tilde{\xi}|^{2} r^{2} d v_{t} d t
\end{aligned}
$$

as $\varepsilon \rightarrow 0$. Here we used (43) and that $\tilde{\xi}$ has compact support.

Furthermore, we obtain also exactly as in the proof of Lemma 3.5 that $\bar{\phi}^{\varepsilon}:=\tilde{\phi}^{\varepsilon}-\hat{\phi}^{\varepsilon}$ converges to zero strongly in $L^{2}\left((0, T) ; H^{1}(\mathbb{T})\right)$, and as a consequence the mixed term vanishes in the limit.

Step 2: Construction for general $\tilde{\xi}$.

In order to finish the proof of the lemma we have to show that we can approximate $\tilde{\xi} \in L^{2}\left(r^{2} d v_{t} d t\right)^{3}$ by smooth functions $\tilde{\xi}_{n}$ with compact support such that

$$
\int_{0}^{T} \beta \int\left|\tilde{\xi}_{n}\right|^{2} r^{2} d v_{t} d t \rightarrow \int_{0}^{T} \beta \int|\tilde{\xi}|^{2} r^{2} d v_{t} d t
$$

as $n \rightarrow \infty$. But this follows from a density argument. 
Proof of Lemma 3.1 We first prove (38). Set $l=\gamma \varepsilon$, where $\gamma$ is as in (51). Due to the fact that $\left\{B_{l}\left(X_{i}\right)\right\}_{i}$ are disjoint we have

$$
\int_{\mathbb{T}}\left|\nabla \mu^{\varepsilon}\right|^{2} d x \geq \sum_{i} \int_{B_{l}\left(X_{i}\right)}\left|\nabla \mu^{\varepsilon}\right|^{2} d x .
$$

On the other hand, for each $i$,

$$
\int_{B_{l}\left(X_{i}\right)}\left|\nabla \mu^{\varepsilon}\right|^{2} d x \geq \inf _{\psi} \int_{B_{l}\left(X_{i}\right)}|\psi|^{2} d x,
$$

where the infimum is taken over all $\psi \in\left(L^{2}\left(B_{l}\left(X_{i}\right)\right)\right)^{2}$ which satisfy

$$
\int_{B_{l}\left(X_{i}\right)} \psi \cdot \nabla \zeta d x=\int_{B_{l}\left(X_{i}\right)}\left(\frac{\varepsilon^{2}}{\alpha_{\varepsilon}^{2}} \chi_{B_{i}}-\pi\right) \zeta d x
$$

for all $\zeta \in C_{0}^{\infty}\left(B_{l}\left(X_{i}\right)\right)$. We see that the minimizer $\hat{\psi}$ is orthogonal to all divergence-free vector-valued functions. Hence $\hat{\psi}=\nabla \phi, \phi=$ const. on $\partial B_{l}\left(X_{i}\right)$, where $-\Delta \phi=\frac{\varepsilon^{2}}{\alpha_{\varepsilon}^{2}} \chi_{B_{i}}-\pi$. We find that $\hat{\psi}=\nabla \mu_{i}$, where $\mu_{i}$ is defined as in (70), and we have

$$
\begin{aligned}
\int_{B_{l}\left(X_{i}\right)}|\hat{\psi}|^{2} d x & =\int_{B_{l}\left(X_{i}\right)}\left|\nabla \mu_{i}\right|^{2} d x \\
& \geq \int_{B_{l}\left(X_{i}\right) \backslash B_{i}} \frac{\left(\varepsilon R_{i}\right)^{4}}{4\left|x-X_{i}\right|^{2}}-\frac{\pi \varepsilon^{2} R_{i}^{2}}{2} d x \\
& =2 \pi \frac{\left(\varepsilon R_{i}\right)^{4}}{4} \ln \frac{l}{\alpha_{\varepsilon} R_{i}}-\frac{\pi^{2}}{2}\left(l^{2}-\left(\alpha_{\varepsilon} R_{i}\right)^{2}\right)\left(\varepsilon R_{i}\right)^{2} .
\end{aligned}
$$

Then it follows from (34), $\sum_{i} \varepsilon^{2} R_{i}^{2}=1$ and (83) that

$$
\sum_{i} \varepsilon^{2} R_{i}^{4}(t) \leq C \text { for all } t \in[0, T]
$$

which proves (38).

Proof of Lemma 3.10 We can prove this in a similar way as in [14, section 3.4] by making use of (32) and Lemma A.1 although we are using Neumann boundary condition on $\partial B_{i}$ instead of Dirichlet boundary condition.

Note $0<\frac{1}{1-\varepsilon^{2} \ln R_{i}} \leq \frac{1}{1+\varepsilon^{2} \ln \varepsilon} \leq \frac{2 e}{2 e-1}$ by $0<R_{i} \leq 1 / \varepsilon$ and $\left|B_{l}\left(X_{i}\right)\right| /\left|A_{i}\right| \leq 2$ for small $\varepsilon$ with $l=\gamma \varepsilon$ and

$$
A_{i}=\left\{x \in \mathbb{T} ; \alpha_{\varepsilon} R_{i}<\left|x-R_{i}\right|<\gamma \varepsilon\right\} .
$$

Setting $v^{\varepsilon}=u^{\varepsilon}+\phi^{\varepsilon}-2 \sigma \mu^{\varepsilon}-\lambda^{\varepsilon}$, we have

$$
\begin{aligned}
& \int \frac{1}{1-\varepsilon^{2} \ln r} \frac{1}{r^{2}} d v_{t}^{\varepsilon}=\sum_{i} \frac{\varepsilon^{2}}{1-\varepsilon^{2} \ln R_{i}} \frac{1}{R_{i}^{2}} \\
& \leq C \sum_{i} \frac{\varepsilon^{2}}{1-\varepsilon^{2} \ln R_{i}}\left|\frac{1}{R_{i}}-\frac{1}{\left|A_{i}\right|} \int_{A_{i}} v^{\varepsilon} d x\right|^{2}+C \sum_{i} \frac{\varepsilon^{2}}{1-\varepsilon^{2} \ln R_{i}}\left|\frac{1}{\left|A_{i}\right|} \int_{A_{i}} v^{\varepsilon} d x\right|^{2} \\
& \leq C \sum_{i} \frac{\varepsilon^{2}}{1-\varepsilon^{2} \ln R_{i}}\left|\frac{1}{R_{i}}-\frac{1}{\left|A_{i}\right|} \int_{A_{i}} v^{\varepsilon} d x\right|^{2}+C \sum_{i} \frac{1}{\left|A_{i}\right|}\left|\int_{A_{i}} v^{\varepsilon} d x\right|^{2}
\end{aligned}
$$


since $\left|B_{l}\left(X_{i}\right)\right| /\left|A_{i}\right| \leq 2$ for small $\varepsilon$. Using

$$
\left|\int_{A_{i}} v^{\varepsilon} d x\right|^{2} \leq\left|A_{i}\right| \int_{A_{i}}\left(v^{\varepsilon}\right)^{2} d x
$$

we see that

$$
\int \frac{1}{1-\varepsilon^{2} \ln r} \frac{1}{r^{2}} d v_{t}^{\varepsilon} \leq C \int_{\mathbb{T}}\left|v^{\varepsilon}\right|^{2} d x+C \sum_{i} \frac{\varepsilon^{2}}{1-\varepsilon^{2} \ln R_{i}}\left|\frac{1}{R_{i}}-\frac{1}{\left|A_{i}\right|} \int_{A_{i}} v^{\varepsilon} d x\right|^{2} .
$$

Now we estimate the second term of the right hand side of (85). Note that $v^{\varepsilon}=u^{\varepsilon}+\phi^{\varepsilon}-$ $2 \sigma \mu^{\varepsilon}-\lambda^{\varepsilon}$ satisfies

$$
\int_{\partial B_{i}} v^{\varepsilon}-\frac{1}{R_{i}} d S=0
$$

for each $i$. Using Lemma A.1 for $f=v^{\varepsilon}-\frac{1}{R_{i}}$, we have

$$
\left|\frac{1}{\left|A_{i}\right|} \int_{A_{i}}\left(\frac{1}{R_{i}}-v^{\varepsilon}\right) d x\right|^{2} \leq C \frac{\left|B_{l}\left(X_{i}\right)\right|^{2}}{\left|A_{i}\right|^{2}} \ln \left(\frac{\gamma \varepsilon}{\alpha_{\varepsilon} R_{i}}\right) \int_{A_{i}}\left|\nabla v^{\varepsilon}\right|^{2} d x,
$$

and since $\left|B_{l}\left(X_{i}\right)\right| /\left|A_{i}\right| \leq 2$ for small $\varepsilon$,

$$
\sum_{i} \frac{\varepsilon^{2}}{1-\varepsilon^{2} \ln R_{i}}\left|\frac{1}{R_{i}}-\frac{1}{\left|A_{i}\right|} \int_{A_{i}} v^{\varepsilon} d x\right|^{2} \leq C \int_{\mathbb{T}}\left|\nabla v^{\varepsilon}\right|^{2} d x .
$$

It follows from (85) and (86) that

$$
\int \frac{1}{1-\varepsilon^{2} \ln r} \frac{1}{r^{2}} d v_{t}^{\varepsilon} \leq C\left(\int_{\mathbb{T}}\left|v^{\varepsilon}\right|^{2} d x+\int_{\mathbb{T}}\left|\nabla v^{\varepsilon}\right|^{2} d x\right) .
$$

We estimate the $L^{2}$ norm of $v^{\varepsilon}$.

$$
\begin{aligned}
\int_{\mathbb{T}}\left|v^{\varepsilon}\right|^{2} d x & \leq C \int_{\mathbb{T}}\left|v^{\varepsilon}-\int_{\mathbb{T}} v^{\varepsilon}\right|^{2} d x+C\left|\int_{\mathbb{T}} v^{\varepsilon} d x\right|^{2} \\
& \leq C \int_{\mathbb{T}}\left|\nabla v^{\varepsilon}\right|^{2} d x+C\left|\int_{\mathbb{T}} v^{\varepsilon} d x\right|^{2}
\end{aligned}
$$

Using

$$
\begin{aligned}
|| A_{i}\left|\int_{\mathbb{T}} v^{\varepsilon} d x\right| & \leq\left|\int_{A_{i}}\left(\int_{\mathbb{T}} v^{\varepsilon}-v^{\varepsilon}\right)\right|+\left|\int_{A_{i}} v^{\varepsilon}\right| \\
& \leq \int_{A_{i}}\left|\int_{\mathbb{T}} v^{\varepsilon}-v^{\varepsilon}\right|+\left|\int_{A_{i}} v^{\varepsilon}\right| \\
& \leq C \varepsilon\left[\int_{A_{i}}\left|\int_{\mathbb{T}} v^{\varepsilon}-v^{\varepsilon}\right|^{2}\right]^{1 / 2}+\left|\int_{A_{i}} v^{\varepsilon}\right|,
\end{aligned}
$$


we see that

$$
\begin{aligned}
& \left(\int \frac{r}{1-\varepsilon^{2} \ln r} d v_{t}^{\varepsilon}\right)\left|\int_{\mathbb{T}} v^{\varepsilon}\right| \\
& \leq C \sum_{i} \frac{R_{i}}{1-\varepsilon^{2} \ln R_{i}}|| A_{i}\left|\int_{\mathbb{T}} v^{\varepsilon} d x\right| \\
& \leq C\left(\sum_{i} \varepsilon^{2} R_{i}^{2}\right)^{1 / 2}\left(\sum_{i} \int_{A_{i}}\left|\int_{\mathbb{T}} v^{\varepsilon}-v^{\varepsilon}\right|^{2}\right)^{1 / 2}+C \sum_{i} \frac{R_{i}}{1-\varepsilon^{2} \ln R_{i}}\left|\int_{A_{i}} v^{\varepsilon}\right| \\
& \leq C\left(\int_{\mathbb{T}}\left|\nabla v^{\varepsilon}\right|^{2} d x\right)^{1 / 2}+C \sum_{i} \frac{R_{i}}{1-\varepsilon^{2} \ln R_{i}}\left|\int_{A_{i}} v^{\varepsilon}\right| .
\end{aligned}
$$

We estimate the second term of the right hand side of (90).

$$
\begin{aligned}
& \sum_{i} \frac{R_{i}}{1-\varepsilon^{2} \ln R_{i}}\left|\int_{A_{i}} v^{\varepsilon}\right| \\
& \quad \leq \sum_{i} \frac{R_{i}\left|A_{i}\right|}{1-\varepsilon^{2} \ln R_{i}}\left|\frac{1}{\left|A_{i}\right|} \int_{A_{i}} v^{\varepsilon}-\frac{1}{R_{i}}\right|+\sum_{i} \frac{\left|A_{i}\right|}{1-\varepsilon^{2} \ln R_{i}} \\
& \quad \leq C\left(\sum_{i} \varepsilon^{2} R_{i}^{2}\right)^{1 / 2}\left(\sum_{i} \frac{\varepsilon^{2}}{1-\varepsilon^{2} \ln R_{i}}\left|\frac{1}{\left|A_{i}\right|} \int_{A_{i}} v^{\varepsilon}-\frac{1}{R_{i}}\right|^{2}\right)^{1 / 2}+C \sum_{i} \varepsilon^{2} \\
& \quad \leq C\left(1+\left(\int_{\mathbb{T}}\left|\nabla v^{\varepsilon}\right|^{2} d x\right)^{1 / 2}\right) .
\end{aligned}
$$

Note that due to

$$
1=\left(\sum_{i} \varepsilon^{2} R_{i}^{2}\right)^{2} \leq\left(\sum_{i} \varepsilon^{2} R_{i}^{3}\left(1-\varepsilon^{2} \ln R_{i}\right)\right)\left(\sum_{i} \frac{\varepsilon^{2} R_{i}}{1-\varepsilon^{2} \ln R_{i}}\right),
$$

$-\ln R_{i} \leq \frac{1}{e \alpha R_{i}^{\alpha}}, \sum_{i} \varepsilon^{4} R_{i}^{2} \leq C$ and $\sum_{i} \varepsilon^{2} R_{i}^{3} \leq C$, there is some $c$, independent of $\varepsilon$, such that

$$
\sum_{i} \frac{\varepsilon^{2} R_{i}}{1-\varepsilon^{2} \ln R_{i}} \geq c>0 .
$$

Then it follows from (90) and (91) that

$$
\left|\int_{\mathbb{T}} v^{\varepsilon}\right| \leq C\left(1+\left(\int_{\mathbb{T}}\left|\nabla v^{\varepsilon}\right|^{2} d x\right)^{1 / 2}\right) .
$$

Then by (88),

$$
\int_{\mathbb{T}}\left|v^{\varepsilon}\right|^{2} d x \leq C\left(\int_{\mathbb{T}}\left|\nabla v^{\varepsilon}\right|^{2} d x+1\right) .
$$

Thus we obtain from (87),

$$
\int \frac{1}{1-\varepsilon^{2} \ln r} \frac{1}{r^{2}} d v_{t}^{\varepsilon} \leq C\left(\int_{\mathbb{T}}\left|\nabla v^{\varepsilon}\right|^{2} d x+1\right) .
$$


Proof of Lemma 3.9 Step 1: An expression for $V_{i}$. Similarly to $\phi_{i}$ in the proof of Lemma 3.4 we introduce a suitable test function $v_{i}$. Here it is the capacity potential of $B_{i}$ with respect to $B_{l}\left(X_{i}\right)$, with $l=\gamma \varepsilon$. This gives that

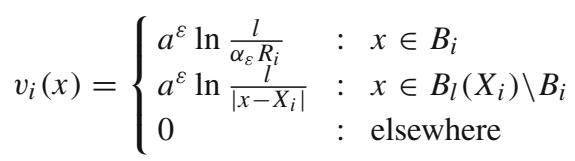

with $a^{\varepsilon}=\left(\ln \frac{l}{\alpha_{\varepsilon} R_{i}}\right)^{-1}$. With this definition we also have

$$
\left[\nabla v_{i} \cdot \vec{n}\right]=-\frac{a^{\varepsilon}}{\alpha_{\varepsilon} R_{i}} \quad \text { on } \partial B_{i} \quad \text { and } \quad\left[\nabla v_{i} \cdot \vec{n}\right]=\frac{a^{\varepsilon}}{l} \quad \text { on } \partial B_{l}\left(X_{i}\right) .
$$

Then

$$
\begin{aligned}
2 \pi R_{i} V_{i} & =2 \pi R_{i} \frac{\alpha_{\varepsilon}}{\varepsilon^{2}}\left[\nabla u^{\varepsilon} \cdot \vec{n}\right] \\
& =\frac{1}{\varepsilon^{2}} \int_{\partial B_{i}}\left[\nabla u^{\varepsilon} \cdot \vec{n}\right] d S \\
& =\frac{1}{\varepsilon^{2}} \int_{\partial B_{i}}\left[\nabla u^{\varepsilon} \cdot \vec{n}\right] v_{i} d S \\
& =\frac{1}{\varepsilon^{2}} \int_{\partial B_{i}}\left[\nabla v_{i} \cdot \vec{n}\right] u^{\varepsilon} d S+\frac{1}{\varepsilon^{2}} \int_{\partial B_{l}\left(X_{i}\right)}\left[\nabla v_{i} \cdot \vec{n}\right] u^{\varepsilon} d S \\
& =-\frac{a^{\varepsilon}}{\varepsilon^{2} \alpha_{\varepsilon} R_{i}} \int_{\partial B_{i}} u^{\varepsilon} d S+\frac{a^{\varepsilon}}{\varepsilon^{2} l} \int_{\partial B_{l}\left(X_{i}\right)} u^{\varepsilon} d S .
\end{aligned}
$$

Due to (32) it follows that

$$
R_{i} V_{i}=-\frac{a^{\varepsilon}}{\varepsilon^{2}}\left(\frac{1}{R_{i}}+\frac{1}{\left|\partial B_{i}\right|} \int_{\partial B_{i}}\left(2 \sigma \mu^{\varepsilon}-\phi^{\varepsilon}\right) d x+\lambda^{\varepsilon}(t)\right)+\frac{a^{\varepsilon}}{2 \pi \varepsilon^{2} l} \int_{\partial B_{l}\left(X_{i}\right)} u^{\varepsilon} d S .
$$

Using that $\mu^{\varepsilon}=\sum_{i} \mu_{i}+\bar{\mu}^{\varepsilon}$ with $\mu_{i}$ as in (70) we obtain

$$
\begin{aligned}
\frac{1}{\left|\partial B_{i}\right|} \int_{\partial B_{i}} \mu^{\varepsilon} d x & =\frac{1}{\left|\partial B_{i}\right|} \int_{\partial B_{i}} \mu_{i} d x+\frac{1}{\left|\partial B_{i}\right|} \int_{\partial B_{i}} \bar{\mu}^{\varepsilon} d x \\
& =\frac{\varepsilon^{2} R_{i}^{2}}{2} \ln \frac{l}{\alpha_{\varepsilon} R_{i}}+O\left(\varepsilon^{2}\right)+\frac{1}{\left|\partial B_{l}\left(X_{i}\right)\right|} \int_{\partial B_{l}\left(X_{i}\right)} \bar{\mu}^{\varepsilon} d x \\
& =\frac{\varepsilon^{2} R_{i}^{2}}{2} \ln \frac{l}{\alpha_{\varepsilon} R_{i}}+O\left(\varepsilon^{2}\right)+\frac{1}{\left|\partial B_{l}\left(X_{i}\right)\right|} \int_{\partial B_{l}\left(X_{i}\right)} \mu^{\varepsilon} d x
\end{aligned}
$$

Similarly using that $\phi^{\varepsilon}=\sum_{i} \phi_{i}+\bar{\phi}^{\varepsilon}$ with $\phi_{i}$ as in (63) we obtain

$$
\begin{aligned}
\frac{1}{\left|\partial B_{i}\right|} \int_{\partial B_{i}} \phi^{\varepsilon} d x & =\frac{1}{\left|\partial B_{i}\right|} \int_{\partial B_{i}} \phi_{i} d x+\frac{1}{\left|\partial B_{i}\right|} \int_{\partial B_{i}} \bar{\phi}^{\varepsilon} d x \\
& =\frac{1}{\left|\partial B_{l}\left(X_{i}\right)\right|} \int_{\partial B_{l}\left(X_{i}\right)} \bar{\phi}^{\varepsilon} d x=\frac{1}{\left|\partial B_{l}\left(X_{i}\right)\right|} \int_{\partial B_{l}\left(X_{i}\right)} \phi^{\varepsilon} d x .
\end{aligned}
$$

It follows that

$$
R_{i} V_{i}=-\frac{a^{\varepsilon}}{\varepsilon^{2}}\left(\frac{1}{R_{i}}+O\left(\varepsilon^{2}\right)+\lambda^{\varepsilon}(t)\right)-\sigma R_{i}^{2}+\frac{a^{\varepsilon}}{2 \pi \varepsilon^{2} l} \int_{\partial B_{l}\left(X_{i}\right)} u^{\varepsilon}+\phi^{\varepsilon}-2 \sigma \mu^{\varepsilon} d S .
$$

Step 2: A bound on $\int_{\partial B_{l}\left(X_{i}\right)} u^{\varepsilon}+\phi^{\varepsilon}-2 \sigma \mu^{\varepsilon} d S$. 
We define $\psi(x)=\frac{1}{2}\left(l^{2}-\left|x-X_{i}\right|^{2}\right)$ in $B_{l}\left(X_{i}\right)$ such that

$-\Delta \psi=2$ in $B_{l}\left(X_{i}\right), \quad-\nabla \psi=x-X_{i}$ in $B_{l}\left(X_{i}\right) \quad$ and $\nabla \psi \cdot \vec{n}=-l$ on $\partial B_{l}\left(X_{i}\right)$.

Thus, abbreviating $v^{\varepsilon}=u^{\varepsilon}+\phi^{\varepsilon}-2 \sigma \mu^{\varepsilon}$, we have

$$
\begin{aligned}
\frac{1}{l} \int_{\partial B_{l}\left(X_{i}\right)} v^{\varepsilon} d S & =-\frac{1}{l^{2}} \int_{\partial B_{l}\left(X_{i}\right)} v^{\varepsilon} \nabla \psi \cdot \vec{n} d S \\
& =-\frac{1}{l^{2}} \int_{B_{l}\left(X_{i}\right)} \Delta \psi v^{\varepsilon}+\nabla \psi \cdot \nabla v^{\varepsilon} d x \\
& =\frac{3}{l^{2}} \int_{B_{l}\left(X_{i}\right)} v^{\varepsilon}-\frac{1}{l^{2}} \int_{B_{l}\left(X_{i}\right)} \nabla \psi \cdot \nabla v^{\varepsilon} d x .
\end{aligned}
$$

We multiply (97) by $R_{i}^{m} a^{\varepsilon}$ with $m \geq 0$, and sum over $i \in I$ for some $I \subset\left\{i: R_{i}>0\right\}$. Then due to $a^{\varepsilon} \leq 2 \varepsilon^{2}$, we find, using $l=\gamma \varepsilon$ and Hölder's inequality, that

$$
\begin{aligned}
& \left|\sum_{i \in I} R_{i}^{m} \frac{a^{\varepsilon}}{l} \int_{\partial B_{l}\left(X_{i}\right)} v^{\varepsilon} d S\right| \\
& \quad \leq C\left(\sum_{i \in I} R_{i}^{m} \int_{B_{l}\left(X_{i}\right)}\left|v^{\varepsilon}\right|+\sum_{i \in I} R_{i}^{m} \int_{B_{l}\left(X_{i}\right)}\left|\nabla \psi \cdot \nabla v^{\varepsilon}\right| d x\right) \\
& \quad \leq C\left(\sum_{i \in I} R_{i}^{m} l^{5 / 3}\left(\int_{B_{l}\left(X_{i}\right)}\left|v^{\varepsilon}\right|^{6}\right)^{1 / 6}+\sum_{i \in I} R_{i}^{m} l^{2}\left(\int_{B_{l}\left(X_{i}\right)}\left|\nabla v^{\varepsilon}\right|^{2}\right)^{1 / 2}\right) \\
& \quad \leq C\left(\sum_{i \in I} \varepsilon^{2} R_{i}^{6 m / 5}\right)^{5 / 6}\left(\int_{\mathbb{T}}\left|v^{\varepsilon}\right|^{6}\right)^{1 / 6}+C\left(\sum_{i \in I} \varepsilon^{4} R_{i}^{2 m}\right)^{1 / 2}\left(\int_{\mathbb{T}}\left|\nabla v^{\varepsilon}\right|^{2}\right)^{1 / 2} .
\end{aligned}
$$

Due to Sobolev embedding and Poincare's inequality (recall that $v^{\varepsilon}$ has mean value zero) we finally find

$$
\begin{aligned}
& \left|\sum_{i \in I} R_{i}^{m} \frac{a^{\varepsilon}}{l} \int_{\partial B_{l}\left(X_{i}\right)} u^{\varepsilon}+\phi^{\varepsilon}-2 \sigma \mu^{\varepsilon} d S\right| \\
& \quad \leq C\left(\int_{\mathbb{T}}\left|\nabla \mu^{\varepsilon}\right|^{2}+\left|\nabla \phi^{\varepsilon}\right|^{2}+\left|\nabla u^{\varepsilon}\right|^{2}\right)^{1 / 2}\left\{\left(\sum_{i \in I} \varepsilon^{2} R_{i}^{6 m / 5}\right)^{5 / 6}+\left(\sum_{i \in I} \varepsilon^{4} R_{i}^{2 m}\right)^{1 / 2}\right\} .
\end{aligned}
$$

Step 3: A bound for $\lambda^{\varepsilon}(t)$. We go back to (96) to estimate $\lambda^{\varepsilon}(t)$. For that purpose we multiply (96) by $\varepsilon^{2}$ and sum over $i$. We find

$$
\begin{aligned}
0 & =-\sum_{i} a^{\varepsilon}\left(\frac{1}{R_{i}}+O\left(\varepsilon^{2}\right)\right)-\sigma \sum_{i} \varepsilon^{2} R_{i}^{2} \\
& -\lambda^{\varepsilon}(t) \sum_{i} a^{\varepsilon}+\sum_{i} \frac{a^{\varepsilon}}{2 \pi l} \int_{\partial B_{l}\left(X_{i}\right)} u^{\varepsilon}+\phi^{\varepsilon}-2 \sigma \mu^{\varepsilon} d S .
\end{aligned}
$$

First notice that due to

$$
\begin{aligned}
& 1=\left(\sum_{i} \varepsilon^{2} R_{i}^{2}(t)\right)^{5} \leq\left(\sum_{i} \varepsilon^{2}\right)\left(\sum_{i} \varepsilon^{4} R_{i}^{4} \ln \frac{l}{\alpha_{\varepsilon} R_{i}}\right)^{2}\left(\sum_{i} \frac{R_{i}}{\ln \frac{l}{\alpha_{\varepsilon} R_{i}}}\right)^{2} \\
& 1=\left(\sum_{i} \varepsilon^{2} R_{i}^{2}(t)\right)^{2} \leq\left(\sum_{i} \varepsilon^{4} R_{i}^{4} \ln \frac{l}{\alpha_{\varepsilon} R_{i}}\right)\left(\sum_{i} \frac{1}{\ln \frac{l}{\alpha_{\varepsilon} R_{i}}}\right)
\end{aligned}
$$


we have $\left(\sum_{i} \frac{1}{\ln \frac{l}{\alpha_{\varepsilon} R_{i}}}\right)^{-1} \leq \sum_{i} \varepsilon^{4} R_{i}^{4} \ln \frac{l}{\alpha_{\varepsilon} R_{i}} \leq C$ due to (84). As a consequence, we also have $\frac{1}{\sum_{i} a^{\varepsilon}} \leq C$.

Taking $m=0$ in (98), we find

$$
\begin{aligned}
\left|\lambda^{\varepsilon}(t)\right| & \leq C\left(\sum_{i} \frac{a^{\varepsilon}}{R_{i}}+\sum_{i} \varepsilon^{2}\left(\varepsilon^{2}+R_{i}^{2}\right)+\left(\int_{\mathbb{T}}\left|\nabla u^{\varepsilon}\right|^{2}+\left|\nabla \mu^{\varepsilon}\right|^{2}+\left|\nabla \phi^{\varepsilon}\right|^{2}\right)^{1 / 2}\right) \\
& \leq C\left(1+\sum_{i} \frac{a^{\varepsilon}}{R_{i}}+\left(\int_{\mathbb{T}}\left|\nabla u^{\varepsilon}\right|^{2}+\left|\nabla \mu^{\varepsilon}\right|^{2}+\left|\nabla \phi^{\varepsilon}\right|^{2}\right)^{1 / 2}\right) .
\end{aligned}
$$

Using

$$
\begin{aligned}
\sum_{i} \frac{a^{\varepsilon}}{R_{i}} \leq \sum_{i} \frac{\varepsilon^{2}}{1-\varepsilon^{2} \ln \frac{R_{i}}{\gamma}} \frac{1}{R_{i}} & \leq\left(\sum_{i} \varepsilon^{2}\right)^{1 / 2}\left(\sum_{i} \frac{\varepsilon^{2}}{1-\varepsilon^{2} \ln \frac{R_{i}}{\gamma}} \frac{1}{R_{i}^{2}}\right)^{1 / 2} \\
& \leq C\left(\int \frac{1}{1-\varepsilon^{2} \ln r} \frac{1}{r^{2}} d v_{t}^{\varepsilon}\right)^{1 / 2}
\end{aligned}
$$

and Lemma 3.10, it follows [cf. (81) in Sect. 3.3] that

$$
\int_{0}^{T}\left|\lambda^{\varepsilon}(t)\right| d t \leq C_{T}
$$

Step 4: Completion of Proof. Now we go back to (96), multiply with $\varepsilon^{2} R_{i}^{2}$ and sum, but only over $i$ such that $R_{i} \geq M$. We find

$$
\begin{aligned}
\sum_{R_{i} \geq M} \varepsilon^{2} R_{i}^{3} V_{i}= & \left(-\sum_{R_{i} \geq M} \varepsilon^{2} R_{i}-\lambda^{\varepsilon}(t) \sum_{R_{i} \geq M} \varepsilon^{2} R_{i}^{2}\right. \\
& \left.+\sum_{R_{i} \geq M} \frac{\varepsilon^{2} R_{i}^{2}}{2 \pi l} \int_{\partial B_{l}\left(X_{i}\right)} u^{\varepsilon}+\phi^{\varepsilon}-2 \sigma \mu^{\varepsilon} d S\right)(1+O(\varepsilon))-\sigma \sum_{R_{i} \geq M} \varepsilon^{2} R_{i}^{4} .
\end{aligned}
$$

Here we used $\frac{a^{\varepsilon}}{\varepsilon^{2}}=\frac{1}{1+\varepsilon^{2} \ln \frac{\gamma}{R_{i}}}=1+O(\varepsilon)$ as $\varepsilon \rightarrow 0$ for $i$ such that $R_{i} \geq M, \varepsilon R_{i} \leq 1$ and $M>\gamma$.

The key feature of (102) is that we have a term of the form $-\sum_{R_{i} \geq M} \varepsilon^{2} R_{i}^{4}$ on the right hand side and otherwise terms which converge to zero as $M \rightarrow \infty$ uniformly in $\varepsilon$. Now define

$$
y(t):=\frac{1}{4} \sum_{R_{i} \geq M} \varepsilon^{2} R_{i}^{4}(t)
$$

such that it follows from (102) together with (98) with $m=2$ that

$$
\begin{aligned}
& y^{\prime}(t)+4 \sigma y(t) \leq C\left\{\left(\left|\lambda^{\varepsilon}(t)\right|+1\right) \sum_{R_{i} \geq M} \varepsilon^{2} R_{i}^{2}\right. \\
& \left.+\left(\int_{\mathbb{T}}\left|\nabla u^{\varepsilon}\right|^{2}+\left|\nabla \mu^{\varepsilon}\right|^{2}+\left|\nabla \phi^{\varepsilon}\right|^{2}\right)^{1 / 2}\left(\left(\sum_{R_{i} \geq M} \varepsilon^{2} R_{i}^{12 / 5}\right)^{5 / 6}+\varepsilon\left(\sum_{R_{i} \geq M} \varepsilon^{2} R_{i}^{4}\right)^{1 / 2}\right)\right\} .
\end{aligned}
$$


Now

$$
\begin{aligned}
& \sum_{R_{i} \geq M} \varepsilon^{2} R_{i}^{2} \leq \frac{1}{M^{2}} \sum_{R_{i} \geq M} \varepsilon^{2} R_{i}^{4} \leq \frac{C}{M^{2}}, \\
& \sum_{R_{i} \geq M} \varepsilon^{2} R_{i}^{12 / 5} \leq \frac{1}{M^{8 / 5}} \sum_{R_{i} \geq M} \varepsilon^{2} R_{i}^{4} \leq \frac{C}{M^{8 / 5}} .
\end{aligned}
$$

To estimate the last term on the right-hand side of (103) we recall that we have the simple estimate $R_{i} \leq C \varepsilon^{-1 / 2}$ such that

$$
\begin{aligned}
& \varepsilon\left(\sum_{R_{i} \geq M} \varepsilon^{2} R_{i}^{4}\right)^{1 / 2} \leq \varepsilon^{1 / 2}\left(\sum_{R_{i} \geq M} \varepsilon^{2} R_{i}^{2}\right)^{1 / 2} \leq C \frac{\varepsilon^{1 / 2}}{M}, \\
& \sum_{R_{i} \geq M} \varepsilon^{4} R_{i}^{4}=\varepsilon^{2} \sum_{R_{i} \geq M} \varepsilon^{2} R_{i}^{4} \leq C \frac{\varepsilon}{M^{2}} .
\end{aligned}
$$

Thus, (103)-(107) imply that we have an estimate of the form

$$
y^{\prime}(t)+4 \sigma y(t) \leq C \frac{f(t)}{M^{\delta}} \quad \text { with } \int_{0}^{T}|f(t)| d t \leq C_{T} \quad \text { and some } \delta>0 .
$$

A simple comparison argument now implies that indeed $y(t) \leq C\left(y(0)+M^{-\delta}\right)$ and thus the statement of the lemma follows with (26).

Proof of Lemma 3.7 We use an argument similar to Step 2 of the proof of [13, Lemma 5.4], and give only an outline of the proof. It follows from the definition of $K(59)$ that

$$
\int_{\mathbb{T}} \nabla K \cdot \nabla \zeta d x=2 \pi\left(\int r^{2} \zeta d v_{t}-\int_{\mathbb{T}} \zeta d x\right)=2 \pi \int r^{2} \zeta d v_{t}
$$

for all $\zeta \in \stackrel{\circ}{H}^{1}(\mathbb{T})$. Furthermore since $\Delta \tilde{\mu}^{\varepsilon}=\pi$ in $\mathbb{T} \backslash \cup_{i}\left(\partial B_{l}\left(X_{i}\right) \cup \partial B_{i}\right),\left[\nabla \tilde{\mu}^{\varepsilon} \cdot \vec{n}\right]=0$ on $\partial B_{i}$, and $\left[\nabla \tilde{\mu}^{\varepsilon} \cdot \vec{n}\right]=-\frac{\varepsilon^{2} R_{i}^{2}}{2 l}$ on $\partial B_{l}\left(X_{i}\right)$, we see that $\tilde{\mu}^{\varepsilon}$ solves

$$
\begin{aligned}
\int_{\mathbb{T}} \nabla \tilde{\mu}^{\varepsilon} \cdot \nabla \zeta d x & =\sum_{i} \int_{\partial B_{l}\left(X_{i}\right)}\left[\nabla \tilde{\mu}^{\varepsilon} \cdot \vec{n}\right] \zeta d S-\pi \int_{\mathbb{T}} \zeta d x \\
& =\sum_{i} \int_{\partial B_{l}\left(X_{i}\right)} \frac{l R_{i}^{2}}{2 \gamma^{2}} \zeta d S
\end{aligned}
$$

for all $\zeta \in \stackrel{\circ}{H}^{1}(\mathbb{T})$. We define

$$
\left\langle L_{\varepsilon}(t), \zeta\right\rangle:=\sum_{i} \int_{\partial B_{l}\left(X_{i}\right)} \frac{l R_{i}^{2}}{\gamma^{2}} \zeta d S, \quad\langle L(t), \zeta\rangle:=2 \pi \int r^{2} \zeta d v_{t}
$$

for $\zeta \in \stackrel{\circ}{H}^{1}(\mathbb{T})$. Then $L_{\varepsilon}(t)$ and $L(t)$ are elements of $\stackrel{\circ}{H}^{-1}(\mathbb{T})=\left(\stackrel{\circ}{H}^{1}(\mathbb{T})\right)^{*}$ for all $t \in[0, T]$. Since $\left\langle L_{\varepsilon}(t), \zeta\right\rangle=2 \int_{\mathbb{T}} \nabla \tilde{\mu}^{\varepsilon} \cdot \nabla \zeta d x$ and $\langle L(t), \zeta\rangle=\int_{\mathbb{T}} \nabla K \cdot \nabla \zeta d x$, we only need to prove that $L_{\varepsilon} \rightarrow L$ strongly in $C^{0}\left([0, T] ; \stackrel{\circ}{H}^{-1}(\mathbb{T})\right)$.

In order to prove this, define

$$
\left\langle\tilde{L}_{\varepsilon}(t), \zeta\right\rangle:=\sum_{i} \int_{B_{l}\left(X_{i}\right)} \frac{2 R_{i}^{2}}{\gamma^{2}} \zeta d x .
$$


Then we see that $\tilde{L}_{\varepsilon}(t) \in\left(L^{4}(\mathbb{T})\right)^{*} \cong L^{4 / 3}(\mathbb{T})$ and satisfies $\left\|\tilde{L}_{\varepsilon}(t)\right\|_{L^{4 / 3}} \leq C$ for all $t \in[0, T]$ and small $\varepsilon>0$. Here $L^{2}$ estimate does not work. Furthermore for all $t_{1}, t_{2} \in[0, T]$ we have

$$
\left\|\tilde{L}_{\varepsilon}\left(t_{1}\right)-\tilde{L}_{\varepsilon}\left(t_{2}\right)\right\|_{L^{4 / 3}} \leq C\left|t_{1}-t_{2}\right|^{1 / 2}\left(\mathcal{D}^{\varepsilon}\right)^{1 / 2}
$$

These mean that $\tilde{L}_{\varepsilon} \in C^{0}\left([0, T] ; L^{4 / 3}(\mathbb{T})\right)$ is uniformly bounded and equicontinuous. It follows from the compact embedding of $L^{4 / 3}(\mathbb{T})$ into $\stackrel{\circ}{H}^{-1}(\mathbb{T})$ and the generalized ArzelaAscoli Theorem that $\tilde{L}_{\varepsilon}$ is relatively compact in $C^{0}\left([0, T] ; \stackrel{\circ}{H}^{-1}(\mathbb{T})\right)$.

Since we can deduce that $\tilde{L}_{\varepsilon}(t) \rightarrow L(t)$ weakly in $\left(C_{p}^{0}\right)^{*}$ for each $t \in[0, T]$, and that $L_{\varepsilon}-\tilde{L}_{\varepsilon} \rightarrow 0$ in $C^{0}\left([0, T] ; \stackrel{\circ}{H}^{-1}(\mathbb{T})\right)$, the proof of Lemma is completed.

Proof of Lemma 3.8 The differential of the nonlocal energy is given by

$$
\begin{aligned}
\left\langle D E_{\mathrm{nl}, \varepsilon}\left(\mathbf{Y}^{\varepsilon}\right), \tilde{\mathbf{Z}}^{\varepsilon}\right\rangle & =-2 \int_{\mathbb{T}} \nabla \mu^{\varepsilon} \cdot \nabla \tilde{w}^{\varepsilon} d x \\
& =-2 \int_{\mathbb{T}} \nabla \mu^{\varepsilon} \cdot\left(\nabla \tilde{u}^{\varepsilon}+\nabla \tilde{\phi}^{\varepsilon}\right) d x
\end{aligned}
$$

with $\tilde{u}^{\varepsilon}$ and $\tilde{\phi}^{\varepsilon}$ determined via (31).

Define $u_{i}$ as in (72), however we take $V_{i}=\tilde{V}_{i}$, and set $\tilde{u}^{\varepsilon}=\sum_{i} u_{i}+\bar{u}^{\varepsilon}$. Note that

$$
\nabla u_{i}=\left\{\begin{array}{cll}
\varepsilon^{2} R_{i} \tilde{V}_{i} \frac{x-X_{i}}{\left|x-X_{i}\right|^{2}} & : & \alpha_{\varepsilon} R_{i}<\left|x-X_{i}\right|<l \\
0 & : & \text { otherwise }
\end{array} .\right.
$$

Also we use the same notations $\tilde{\mu}_{i}, \tilde{\mu}^{\varepsilon}$ as in Sect. 3.4.

We write

$$
\begin{aligned}
& \int_{0}^{T} \beta \int_{\mathbb{T}} \nabla \tilde{u}^{\varepsilon} \cdot \nabla \mu^{\varepsilon} d x d t \\
& =\int_{0}^{T} \beta \int_{\mathbb{T}} \nabla \tilde{u}^{\varepsilon} \cdot\left(\nabla \sum_{j} \tilde{\mu}_{j}+\nabla \frac{K}{2}\right) d x d t+\int_{0}^{T} \beta \int_{\mathbb{T}} \nabla \tilde{u}^{\varepsilon} \cdot \nabla\left(\tilde{\mu}^{\varepsilon}-\frac{K}{2}\right) d x d t \\
& =-\int_{0}^{T} \beta \sum_{i} \int_{\partial B_{i}} \frac{\varepsilon^{2} \tilde{V}_{i}}{\alpha_{\varepsilon}} \sum_{j} \tilde{\mu}_{j} d S d t-\int_{0}^{T} \frac{\beta}{2} \sum_{i} \int_{B_{l}\left(X_{i}\right)} \nabla u_{i} \cdot \nabla K d x d t \\
& \quad+\int_{0}^{T} \frac{\beta}{2} \int_{\mathbb{T}} \nabla \bar{u}^{\varepsilon} \cdot \nabla K d x d t+\int_{0}^{T} \beta \int_{\mathbb{T}} \nabla \tilde{u}^{\varepsilon} \cdot \nabla\left(\tilde{\mu}^{\varepsilon}-\frac{K}{2}\right) d x d t .
\end{aligned}
$$

It follows from the condition (60) that

$$
(1-o(1)) 4 \pi \int_{0}^{T} \sum_{i} \varepsilon^{2} R_{i}^{2} \tilde{V}_{i}^{2} d t \leq \int_{0}^{T} \int_{\mathbb{T}}\left|\nabla \tilde{u}^{\varepsilon}\right|^{2} d x d t \leq C
$$

and that $\bar{u}^{\varepsilon}$ converges to $\tilde{u}$ strongly in $L^{2}((0, T) \times \mathbb{T})$, where $\tilde{u}$ is determined via (53). (See the proof of [13, Lemma 5.4] for details.) Using

$$
\left|\sum_{R_{i} \geq M} \varepsilon^{2} R_{i}^{3} \tilde{V}_{i}\right| \leq\left(\sum_{R_{i} \geq M} \varepsilon^{2} R_{i}^{4}\right)^{1 / 2}\left(\sum_{i} \varepsilon^{2} R_{i}^{2} \tilde{V}_{i}^{2}\right)^{1 / 2}
$$


(113), Lemma 3.9, $\left|R_{i} \ln 1 / R_{i}\right| \leq C$ for $R_{i} \ll 1$ and $\varepsilon R_{i} \leq 1$, we see that

$$
\begin{aligned}
-\int_{0}^{T} \beta \sum_{i} \int_{\partial B_{i}} \frac{\varepsilon^{2} \tilde{V}_{i}}{\alpha_{\varepsilon}} \sum_{j} \tilde{\mu}_{j} d S d t & =-\int_{0}^{T} \beta \sum_{i} \int_{\partial B_{i}} \frac{\varepsilon^{2} \tilde{V}_{i}}{\alpha_{\varepsilon}} \tilde{\mu}_{i} d S d t \\
= & -\int_{0}^{T} \beta \sum_{i} \pi \varepsilon^{4} R_{i}^{3} \tilde{V}_{i} \ln \frac{l}{\alpha_{\varepsilon} R_{i}} d t \\
& \rightarrow-\pi \int_{0}^{T} \beta \int r^{3} \tilde{v} d v_{t} d t
\end{aligned}
$$

as $\varepsilon \rightarrow 0$. In addition, we obtain

$$
\begin{aligned}
\lim _{\varepsilon \rightarrow 0} \int_{0}^{T} \beta \int_{\mathbb{T}} \nabla \bar{u}^{\varepsilon} \cdot \nabla K d x d t & =\int_{0}^{T} \beta \int_{\mathbb{T}} \nabla \tilde{u} \cdot \nabla K d x d t \\
& =-2 \pi \int_{0}^{T} \beta \int r K(t, x) \tilde{v} d v_{t} d t
\end{aligned}
$$

and it follows from Lemma 3.7 that

$$
\lim _{\varepsilon \rightarrow 0} \int_{0}^{T} \beta \int_{\mathbb{T}} \nabla \tilde{u}^{\varepsilon} \cdot \nabla\left(\tilde{\mu}^{\varepsilon}-\frac{K}{2}\right) d x d t=0 .
$$

Finally from $\sum_{i}\left|B_{l}\left(X_{i}\right)\right| \leq C \gamma^{2} \sum_{i} \varepsilon^{2} \leq C \gamma^{2}$ and (111),

$$
\begin{aligned}
\left|\sum_{i} \int_{B_{l}\left(X_{i}\right)} \nabla u_{i} \cdot \nabla K d x\right| & \leq \sum_{i}\left(\int_{B_{l}\left(X_{i}\right)}\left|\nabla u_{i}\right|^{2} d x\right)^{1 / 2}\left(\int_{B_{l}\left(X_{i}\right)}|\nabla K|^{2} d x\right)^{1 / 2} \\
& \leq\left(\sum_{i} \int_{B_{l}\left(X_{i}\right)}\left|\nabla u_{i}\right|^{2} d x\right)^{1 / 2}\left(\sum_{i} \int_{B_{l}\left(X_{i}\right)}|\nabla K|^{2} d x\right)^{1 / 2} \\
& \leq C \omega\left(C \gamma^{2}\right),
\end{aligned}
$$

where

$$
\omega(z):=\sup _{|E| \leq z}\left(\int_{E}|\nabla K|^{2} d x\right)^{1 / 2} .
$$

Since we can choose $\gamma$ arbitrarily small, we obtain

$$
\lim _{\varepsilon \rightarrow 0} \int_{0}^{T} \beta \int_{\mathbb{T}} \nabla \tilde{u}^{\varepsilon} \cdot \nabla \mu^{\varepsilon} d x d t=-\pi \int_{0}^{T} \beta \int\left(r^{3}+r K(x)\right) \tilde{v} d v_{t} d t
$$

from (112), (114), (115), (116) and (117).

Similarly we can prove that $\int_{0}^{T} \beta \int_{\mathbb{T}} \nabla \tilde{\phi}^{\varepsilon} \cdot \nabla \mu^{\varepsilon} d x d t \rightarrow 0$ as $\varepsilon \rightarrow 0$. This completes the proof.

Acknowledgements Open Access funding provided by Projekt DEAL.

Open Access This article is licensed under a Creative Commons Attribution 4.0 International License, which permits use, sharing, adaptation, distribution and reproduction in any medium or format, as long as you give appropriate credit to the original author(s) and the source, provide a link to the Creative Commons licence, and indicate if changes were made. The images or other third party material in this article are included in the article's Creative Commons licence, unless indicated otherwise in a credit line to the material. If material is not included in the article's Creative Commons licence and your intended use is not permitted by statutory regulation or exceeds the permitted use, you will need to obtain permission directly from the copyright holder. To view a copy of this licence, visit http://creativecommons.org/licenses/by/4.0/. 


\section{A Appendix}

Lemma A.1 $A=\left\{x \in \mathbb{R}^{2} ; a<|x|<b\right\}$. There holds

$$
\left|\frac{1}{\pi b^{2}} \int_{A} f d x\right|^{2} \leq \frac{1}{2 \pi}\left(\ln \frac{b}{a}\right) \int_{A}|\nabla f|^{2} d x
$$

for all $f \in H^{1}(A)$ such that $\int_{\partial B_{a}} f d S=0$.

Proof Using the polar coordinate $(r, \theta)$, we write $f=f(r, \theta)$. Let

$$
g(r)=\int_{0}^{2 \pi} f(r, \theta) d \theta
$$

for $r \in[a, b]$. Then by

$$
g^{\prime}(s)=\int_{0}^{2 \pi} f_{r}(s, \theta) d \theta
$$

and

$$
g^{\prime}(s)^{2} \leq 2 \pi \int_{0}^{2 \pi} f_{r}(s, \theta)^{2} d \theta
$$

for $s \in[a, b]$, we get

$$
\int_{a}^{b} g^{\prime}(s)^{2} s d s \leq 2 \pi \int_{a}^{b} s \int_{0}^{2 \pi} f_{r}(s, \theta)^{2} d \theta d s \leq 2 \pi\|\nabla f\|_{2}^{2} .
$$

Note that $g(r)=\int_{a}^{r} g^{\prime}(s) d s$ by $g(a)=0$. Hence

$$
\begin{aligned}
|g(r)| & =\left|\int_{a}^{r} g^{\prime}(s) d s\right| \leq\left(\int_{a}^{b} g^{\prime}(s)^{2} s d s\right)^{1 / 2}\left(\int_{a}^{r} \frac{1}{s} d s\right)^{1 / 2} \\
& \leq \sqrt{2 \pi}\|\nabla f\|_{2}\left(\ln \frac{r}{a}\right)^{1 / 2} .
\end{aligned}
$$

We find

$$
\begin{aligned}
\left|\int_{A} f d x\right|=\left|\int_{a}^{b} g(r) r d r\right| & \leq \sqrt{2 \pi}\|\nabla f\|_{2} \int_{a}^{b} r\left(\ln \frac{r}{a}\right)^{1 / 2} d r \\
& \leq \sqrt{2 \pi}\|\nabla f\|_{2} \frac{b^{2}}{2}\left(\ln \frac{b}{a}\right)^{1 / 2} .
\end{aligned}
$$

Remark: Similarly we can also show that

$$
\lim _{\varepsilon \rightarrow 0} E\left(\mathbf{Y}^{\varepsilon}\right)=E\left(v_{t}\right), \quad \text { uniformly in } t \in[0, T],
$$

where

$$
E\left(v_{t}\right)=2 \pi \int\left(r+\sigma \frac{r^{4}}{4}\right) d v_{t}+\frac{\sigma}{4} \int_{\mathbb{T}}|\nabla K|^{2} d x .
$$

This statement is of interest in itself, but since we do not need it here we omit the proof for the sake of brevity. 


\section{References}

1. Alberti, G., Choksi, R., Otto, F.: Uniform energy distribution for an isoperimetric problem with long-range interactions. J. Am. Math. Soc. 22-2, 569-605 (2009)

2. Bates, F.S., Fredrickson, G.H.: Block copolymers-designer soft materials. Phys. Today 52-2, 32-38 (1999)

3. Chen, X., Oshita, Y.: Periodicity and uniqueness of global minimizers of an energy functional containing a long-range interaction. SIAM J. Math. Anal. 37, 1299-1332 (2005)

4. Chen, X., Oshita, Y.: An application of the modular function in nonlocal variational problems. Arch. Ration. Mech. Anal. 186-1, 109-132 (2007)

5. Escher, J., Nishiura, Y.: Smooth unique solutions for a modified Mullins-Sekerka model arising in diblock copolymer melts. Hokkaido Math. J. 31-1, 137-149 (2002)

6. Glasner, K., Choksi, R.: Coarsening and self-organization in dilute diblock copolymer melts and mixtures. Physica D 238, 1241-1255 (2009)

7. Fife, P., Hilhorst, D.: The Nishiura-Ohnishi free boundary problem in the 1D case. SIAM J. Math. Anal. 33, 589-606 (2001)

8. Hamley, I.W.: The Physics of Block Copolymers. Oxford Science Publications, Oxford (1998)

9. Henry, M., Hilhorst, D., Nishiura, Y.: Singular limit of a second order nonlocal parabolic equation of conservative type arising in the micro-phase separation of diblock copolymers. Hokkaido Math. J. 32, 561-622 (2003)

10. Helmers, M., Niethammer, B., Ren, X.: Evolution in off-critical diblock-copolymer melts. Netw Heterog Media 3-3, 615-632 (2008)

11. Le, N.Q.: On the convergence of the Ohta-Kawasaki evolution equation to motion by Nishiura-Ohnishi law. Preprint (2009)

12. Niethammer, B., Oshita, Y.: A rigorous derivation of mean-field models for diblock copolymer melts. Calc. Var. PDE 39, 273-305 (2010)

13. Niethammer, B., Otto, F.: Ostwald ripening: the screening length revisited. Calc. Var. PDE 13-1, 33-68 (2001)

14. Niethammer, B., Otto, F.: Domain coarsening in thin films. Commun. Pure Appl. Math. 54-3, 361-384 (2001)

15. Nishiura, Y., Ohnishi, I.: Some aspects of the micro-phase separation in diblock copolymers. Physica D 84, 31-39 (1995)

16. Nishiura, Y., Suzuki, H.: Higher dimensional SLEP equation and applications to morphological stability in polymer problems. SIAM J. Math. Anal. 36-3, 916-966 (2004/05)

17. Ohnishi, I., Nishiura, Y., Imai, M., Matsushita, Y.: Analytical solutions describing the phase separation driven by a free energy functional containing a long-range interaction term. CHAOS 9-2, 329-341 (1999)

18. Ohta, T., Kawasaki, K.: Equilibrium morphology of block copolymer melts. Macromolecules 19, 26212632 (1986)

19. Ren, X., Wei, J.: Concentrically layered energy equilibria of the di-block copolymer problem. Eur. J. Appl. Math. 13, 479-496 (2002)

20. Ren, X., Wei, J.: On energy minimizers of the diblock copolymer problem. Interfaces Free Bound. 5, 193-238 (2003)

21. Ren, X., Wei, J.: On the spectra of three-dimensional lamellar solutions of the Diblock copolymer problem. SIAM J. Math. Anal. 35, 1-32 (2003)

Publisher's Note Springer Nature remains neutral with regard to jurisdictional claims in published maps and institutional affiliations. 